\title{
The herpetofauna of Parque Nacional da Serra das Confusões, state of Piauí, Brazil, with a regional species list from an ecotonal area of Cerrado and Caatinga
}

\author{
Francisco Dal Vechio ${ }^{1,3}$, Mauro Teixeira Jr. ${ }^{1}$, Renato Sousa Recoder ${ }^{1}$, Miguel Trefault Rodrigues ${ }^{1}$ \\ \& Hussam Zaher ${ }^{2}$ \\ ${ }^{1}$ Universidade de São Paulo, Instituto de Biociências, Departamento de Zoologia, Rua do Matão, Travessa 14, \\ 321, CEP 05508-090, São Paulo, SP, Brazil. \\ ${ }^{2}$ Museu de Zoologia da Universidade de São Paulo, São Paulo, SP, Brazil. \\ ${ }^{3}$ Corresponding author: Francisco Dal Vechio, e-mail: francisco.dalvechio@gmail.com
}

DAL VECHIO, F., TEIXEIRA JR., M., RECODER, R.S., RODRIGUES, M.T., ZAHER, H. The herpetofauna of Parque Nacional da Serra das Confusões, state of Piauí, Brazil, with a regional species list from an ecotonal area of Cerrado and Caatinga. Biota Neotropica. 16(3): e20150105. http://dx.doi.org/10.1590/16760611-BN-2015-0105

\begin{abstract}
Different physiognomies at Parque Nacional da Serra das Confusões (PNSCo) were intensively sampled aiming to access the distribution pattern of its herpetofauna. Sixty six species were found in the park (47 reptiles and 19 amphibians); the rarefaction curve for lizards, although not fully stabilized in an asymptote, indicates that the sampling effort was enough to reveal most lizard species occurring in the area; and richness estimators recovered values close to observed. For amphibians, the curve shows a weak tendency to stabilization with richness estimators indicating that additional records could be done. Field work carried out at PNSCo has highlighted an unique herpetofauna: five new species were described and there are three candidates as new species. The regional list including Cerrados's units - Estação Ecológica Serra Geral do Tocantins (EESGT) and Estação Ecológica de Uruçuí-Una (EEUU) with Caatinga's ones - PNSCo and Parque Nacional da Serra da Capivara (PNSCa), shows a high herpetofaunal diversity (191 species) to the region. The cluster analysis recovered the Cerrados's units and Caatinga's ones, in separate clusters evidencing a species turnover between domains, despite its geographical proximity. Thus, although there is widespread fauna throughout region shared by the units, each reserve holds its own faunal identity, harboring a singular assemblage of species.
\end{abstract}

Keywords: Reptiles, Amphibians, Herpetofaunal Inventory, Herpetofaunistic similarities.

DAL VECHIO, F., TEIXEIRA JR., M., RECODER, R.S., RODRIGUES, M.T., ZAHER, H. A herpetofauna do Parque Nacional da Serra das Confusões, Piauí, Brasil, com uma lista regional para uma área ecotonal entre o Cerrado e a Caatinga. Biota Neotropica. 16(3): e20150105. http://dx.doi.org/10.1590/1676-0611-BN-2015-0105

Resumo: Diferentes fisionomias foram intensivamente amostradas no Parque Nacional da Serra das Confusões (PNSCo) com o objetivo de acessar o padrão de distribuição da sua herpetofauna. Sessenta e seis espécies foram encontradas no parque (47 répteis e 19 anfíbios); a curva de rarefação de lagartos, embora não totalmente estabilizada em uma assíntota, indica que o esforço amostral foi suficiente para revelar a maioria das espécies de lagartos que ocorrem na área; e estimadores de riqueza recuperaram valores perto dos observados. Para os anfíbios, a curva mostra uma fraca tendência para a estabilização com estimadores de riqueza indicando que registros adicionais podem ser feitos. O trabalho de campo realizado no PNSCo destacou uma herpetofauna única: cinco novas espécies foram descritas e há três candidatos como novas espécies. A lista regional, incluindo unidades de Cerrado - Estação Ecológica Serra Geral do Tocantins (EESGT) e Estação Ecológica Uruçuí-Una (EEUU) com aqueles da Caatinga - PNSCo e Parque Nacional da Serra da Capivara (PNSCa), mostra uma alta diversidade da herpetofauna (191 espécies) para a região. A análise de agrupamento recupera as unidades do Cerrado e as da Caatinga em grupos separados, evidenciando uma substituição de espécies entre os domínios, apesar da sua proximidade geográfica. Assim, embora haja fauna ocorrente em toda a região e compartilhada pelas unidades, cada uma das reservas mantém a sua própria identidade, abrigando um conjunto singular de espécies.

Palavras-chave: Répteis, Anfibios, Inventário da herpetofauna, Similaridade herpetofaunística. 


\section{Introduction}

For many years, our limited knowledge of the herpetofaunal diversity present in the Brazilian Cerrado and Caatinga biomes led us to the erroneous idea these open formation areas were characterized by low endemism rates (Sick 1965, Vanzolini 1963, 1976, 1988). However, extensive field work carried out in last two decades showed the opposite, with elevated local diversity recorded throughout their extension (Cerrado: Colli et al. 2002, Rodrigues 1987, Nogueira et al. 2009, Vitt et al. 2005, França \& Araújo 2006, Silveira 2006, Recoder \& Nogueira 2007, Recoder et al. 2011, Dal Vechio et al. 2013; Caatinga: Borges-Nojosa \& Cascon 2005, Ribeiro et al. 2008, Loebmann \& Haddad 2010, Moura et al. 2011a, Moura et al. 2011b) and exceptional regional diversity along the contact zone between the Cerrado and Caatinga biomes (Rodrigues 1984a,b, 1987, 1988, 1996; Nogueira et al. 2010, Nogueira et al. 2011).

Such efforts also resulted in the description of several new species for the region (Cerrado: Colli et al. 2003, Ferrarezzi et al. 2005, Nogueira \& Rodrigues 2006, Rodrigues et al. 2007, 2008, Pinna et al. 2010, Teixeira Jr. et al. 2012; Arias et al. 2014; Recoder et al. 2014; Caatinga: Rodrigues et al. 2001, Rodrigues et al. 2003b, Bour and Zaher 2005, Nogueira \& Rodrigues 2006, Arias et al. 2011a, Arias et al. 2011b, Passos et al. 2011) and the discovery of large populations of species that were considered rare until now (Rodrigues et al. 2013). High diversity in Cerrado faunal assemblages can be explained by local physiognomic heterogeneity, (Eiten 1972, Oliveira-Filho \& Ratter 2002, Colli et al. 2002, Nogueira et al. 2009) and historical climatic stability (Werneck et al. 2012). The Caatinga domain, on the other hand, presents more homogeneous drier habitats, and a singular fauna that is associated with these phytoecological conditions (Rodrigues 2003a).

Both Cerrado and Caatinga biomes lost extensive areas of original vegetation, mostly due to fast expansion of agricultural enterprises (Myers et al. 2000, Cavalcanti \& Joly 2002, Klink \& Machado 2005, Silva et al. 2006). With this continuous process of habitat loss, it seems imperative to prioritize faunal inventories within both biomes in order to fill the geographical gaps in our knowledge, allowing more accurate decisions when defining areas for conservation (Brooks et al. 1992; Greene 1994; Silva \& Bates 2002). According to Castro (2000), the state of Piauí harbors an important center of biodiversity, mainly for its highly diversified vegetation that accounts for a large ecotonal zone that includes Cerrado, Dry Forest, Caatinga and pre-Amazon elements.

Here, we present a taxonomic list of the local herpetofauna present at the Parque Nacional da Serra das Confusões (PNSCo), located in southwestern Piauí, one of the least studied areas of the Cerrado-Caatinga ecotone. We also provide a regional list of the herpetofauna that includes three intensively sampled conservation units that are geographically closer to PNSCo, as follows: Parque Nacional da Serra da Capivara (PNSCa), Estação Ecológica de Uruçuí-Una (EEUU) and Estação Ecológica Serra Geral do Tocantins (EESGT). Finally, we compare the herpetofaunal composition of PNSCo with other local lists from the Caatinga and neighboring biomes.

\section{Materials and Methods}

\section{Study area}

The Parque Nacional da Serra das Confusões (PNSCo) (approximate coordinates: $08^{\circ} 32^{\prime}-09^{\circ} 16^{\prime} \mathrm{S}, 43^{\circ} 15^{\prime}-43^{\circ} 51^{\prime} \mathrm{W}$, Figures 1, 2) was created in October 1998, and preserves an area of approximately 500.000 ha in southwestern Piauí, close to the border of the state of Bahia. The park is placed in an ecotonal area of Tropical Dry Forest and Caatinga (Olson et al. 2001), with a predominance of deciduous forest and arboreal Caatinga. Climate is considered to be tropical semiarid. The park is situated within the Parnaíba drainage, in a sandstone plateau with altitudes ranging from about 450 to $700 \mathrm{~m}$ above the sea level [a.s.1.]) (Rodrigues et al. 2001; Bour \& Zaher 2005). The plateau is locally known as "Chapada da Serra Grande," contains most of the area belonging to the park, and is dissected on its southwestern portion by the intermittent Santana River drainage (Rodrigues et al., 2001; Bour and Zaher, 2005). The top of the plateau, averages $550 \mathrm{~m}$ a.s.l., being covered by a tall arboreal caatinga that grows on sandy soil and produces an abundant leaf litter. The plateau is dissected by the intermitent Itaueira River in its central portion and by the "Lagoa do Jacu" depression created by erosive processes in its northern portion. The southern and western borders of the park are deeply dissected, with scattered rocky outcrops over sandy soil originated from the erosion of the Chapada da Serra Grande plateau. Lowland areas are characterized by dense rocky outcrops with low arboreal Caatinga growing over a sandy soil. This vegetation is widespread on the open lowlands. Several canyons, dissected by intermittent rivers with an evergreen forest and a dense leaf litter, are also present, but account for only a minor portion of the area.

\section{Sampling method}

Sampling was carried out in two campaigns in the years 2000 and 2002 , one at the end of the dry season (September $26^{\text {th }}$ to October $10^{\text {th }}$ 2000 ) and other during the rainy season (January $10^{\text {th }}$ to $26^{\text {th }} 2002$ ). The main physiognomic subunits present in the region were sampled using pitfall traps with drift fences, complemented by active visual searches. Twelve lines of pitfall traps were installed, each composed of 10 sampling units that consisted of four 30 liters buckets arranged in Y-shape, with a central one connecting to three peripherals by $4 \mathrm{~m}$ long plastic fences. Habitat types, geographic coordinates, campaigns and the total sampling effort are summarized in Table 1. The following habitats were sampled:

1. Dry Forest (Lagoa do Jacu) - Line 1. Mesophitic forest, growing on sandy soil, embedded within the river valley. Dominated by arboreal elements, with canopy reaching about $20 \mathrm{~m}$ and understory with sparse shrubs. Forest with variable width, reaching $25 \mathrm{~m}$ wider sections.

2. High Arboreal Caatinga (Serra Grande Plateau) - Lines 2 to 5. Vegetation on top of the plateau, consisting of arboreal and shrubby elements, characterized by Euphorbiaceae and Leguminosae such as "mimosa" (Anadenanthera macrocarpa). Canopy at about $6 \mathrm{~m}$, growing over sandy soil.

3. High Arboreal Caatinga (Serra das Confusões Plateau) - Lines 6 and 7. Predominantly arboreal vegetation growing over sandy/clay soil, covered with leaf litter. Canopy reaching 15 $\mathrm{m}$ in height with understory undeveloped.

4. Low Arboreal Caatinga (Lowland) - Lines 8 and 9. Sandstone outcrops, covered by xeromorphic vegetation, dominated by Cactaceae, Bromeliaceae and Velloziaceae.

5. Grooved Forest (Lowland) - Line 10. Evergreen forest along canyon bottoms, with great number of bromeliads, pteridophytes and tall trees, around 30-40 m in height, such as jatobá (Hymenaea spp.), forming continuous canopy. 


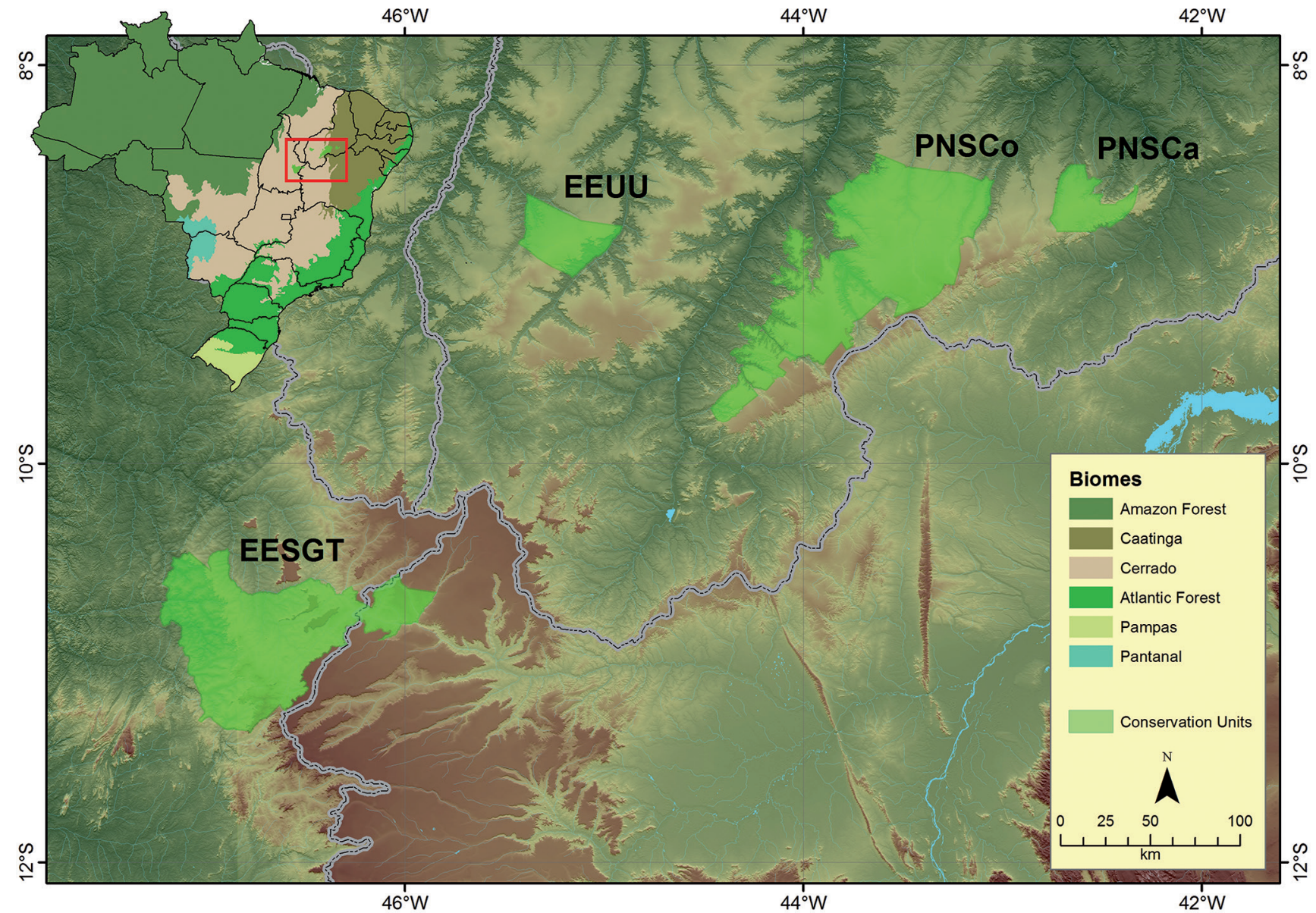

Figure 1. Map of northwestern Brazil showing the conservation units: Estação Ecológica Serra Geral do Tocantins (EESGT), Estação Ecológica de Uruçuí-Una (EEUU) Parque Nacional da Serra das Confusões (PNSCo) and Parque Nacional da Serra da Capivara (PNSCa).

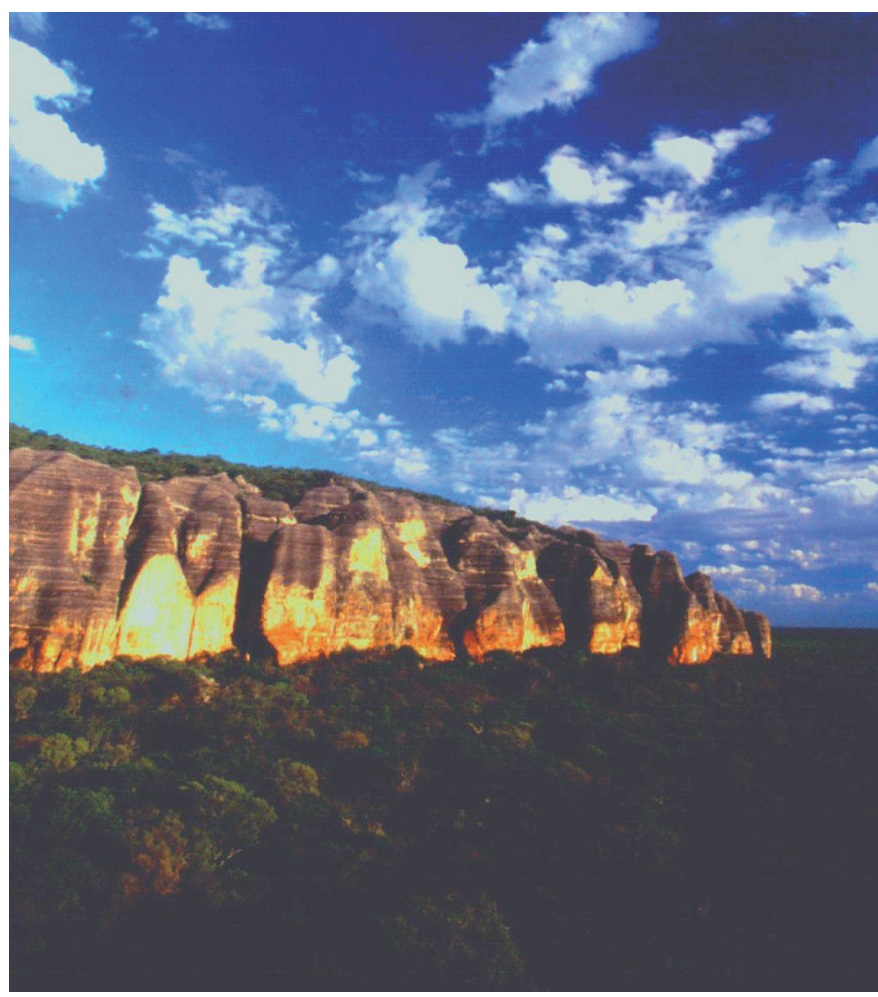

Figure 2. View of plateau with rock outcrop and arboreal caatinga in its base.
It remains constantly in the shade, and accumulates large layers of leaf litter over sandy soil.

6. High arboreal Caatinga (Lowland) - Lines 11 and 12. High arboreal elements, with canopy at $10 \mathrm{~m}$ above sandy soil rich in organic matter and closed understory. This type of vegetation predominates in the valleys.

A representative sample of specimens was collected and housed at the herpetological collection of the Museu de Zoologia, Universidade de São Paulo, São Paulo, Brazil (MZUSP). Voucher specimens were fixed in $10 \%$ formalin and preserved in $70 \%$ alcohol. The remaining individuals were marked by toe clipping and released near the capture site.

\section{Data Analysis}

The effectiveness of the sampling effort was estimated based on rarefaction curves, through 10.000 randomizations without replacement, of a matrix containing the number of individuals per species at each sampling day, including data from both pitfall traps and active search, for lizards and anurans. This analysis was performed using EstimateS v.8.0.0 (Colwell 2006). We also estimated species richness with the Chao 2 and Jacknife 1 estimators. Snakes were excluded from this analysis because capture rates were low, and the resulting list for the group may be underestimated, which could compromise the behavior of the rarefaction curves.

Initially, we performed faunistic comparisons with herpetofaunal lists available for intensively sampled (long-term sampling) conservation 
Table 1. All pitfall lines with its accurate coordinate and the effort spent in each campaign.

\begin{tabular}{|c|c|c|}
\hline Pitfall Lines & $\begin{array}{c}\text { Drier Season } \\
\left(1^{\text {st }} \text { campaign }\right)\end{array}$ & $\begin{array}{c}\text { Wet season } \\
\left(2^{\text {nd }} \text { campaign }\right)\end{array}$ \\
\hline $\begin{array}{l}\text { Line } 1 \text { - Dry Forest (Lagoa do Jacu) } \\
08^{\circ} 40^{\prime} 37^{\prime \prime} \mathrm{S}, 43^{\circ} 29^{\prime} 09^{\prime \prime} \mathrm{W}\end{array}$ & 320 & \\
\hline $\begin{array}{l}\text { Line } 2 \text { - Toca da Cabocla } \\
\text { High Arboreal Caatinga } \\
08^{\circ} 55^{\prime} 28^{\prime \prime} \mathrm{S}, 43^{\circ} 26^{\prime} 58^{\prime \prime} \mathrm{W}\end{array}$ & 400 & \\
\hline $\begin{array}{l}\text { Line } 3 \text { - Morrinhos } \\
\text { High Arboreal Caatinga } \\
08^{\circ} 57^{\prime} 38^{\prime \prime} \mathrm{S}, 43^{\circ} 26^{\prime} 50^{\prime \prime} \mathrm{W}\end{array}$ & 360 & \\
\hline $\begin{array}{l}\text { Line } 4 \text { - Camaçari } \\
\text { High Arboreal Caatinga } \\
08^{\circ} 59^{\prime} 10^{\prime \prime} \mathrm{S}, 43^{\circ} 26^{\prime} 45^{\prime \prime} \mathrm{W}\end{array}$ & & 560 \\
\hline $\begin{array}{l}\text { Line } 5 \text { - Camaçari } \\
\text { High Arboreal Caatinga } \\
08^{\circ} 59^{\prime} 10^{\prime \prime} \mathrm{S}, 43^{\circ} 26^{\prime} 45^{\prime} \mathrm{W}\end{array}$ & & 560 \\
\hline $\begin{array}{l}\text { Line } 6 \text { - Guarita IBAMA } 1 \\
\text { High Arboreal Caatinga } \\
09^{\circ} 13^{\prime} 38^{\prime \prime} \mathrm{S}, 43^{\circ} 27^{\prime} 05^{\prime \prime} \mathrm{W}\end{array}$ & 440 & \\
\hline $\begin{array}{l}\text { Line } 7 \text { - Guarita IBAMA } 2 \\
\text { High Arboreal Caatinga } \\
08^{\circ} 59^{\prime} 10^{\prime \prime} \mathrm{S}, 43^{\circ} 26^{\prime} 45^{\prime \prime} \mathrm{W}\end{array}$ & & 560 \\
\hline $\begin{array}{l}\text { Line } 8 \text { - Olho d'água Santa } \\
\text { Low Arboreal Caatinga (Low Land) } \\
09^{\circ} 13^{\prime} 03^{\prime \prime} \mathrm{S}, 43^{\circ} 29^{\prime} 26^{\prime} \mathrm{W}\end{array}$ & 520 & 600 \\
\hline $\begin{array}{l}\text { Line } 9 \text { - Baixão do Fausto } \\
\text { Low Arboreal Caatinga (Low Land) } \\
09^{\circ} 09^{\prime} 53^{\prime \prime} \mathrm{S}, 43^{\circ} 33^{\prime} 58^{\prime} \text { W }\end{array}$ & & 440 \\
\hline $\begin{array}{l}\text { Line } 10 \text { - Grooved Forest (Low Land) } \\
09^{\circ} 13^{\prime} 10^{\prime \prime} \mathrm{S}, 43^{\circ} 29^{\prime} 27^{\prime \prime} \mathrm{W}\end{array}$ & 400 & \\
\hline $\begin{array}{l}\text { Line } 11 \text { - Pinga Velho } 1 \\
\text { High arboreal Caatinga (Low Land) } \\
09^{\circ} 03^{\prime} 46^{\prime \prime} \mathrm{S}, 43^{\circ} 44^{\prime} 27^{\prime \prime} \mathrm{W}\end{array}$ & & 80 \\
\hline $\begin{array}{l}\text { Line } 12 \text { - Pinga Velho } 2 \\
\text { High arboreal Caatinga (Low Land) } \\
09^{\circ} 03^{\prime} 42^{\prime \prime} \mathrm{S}, 43^{\circ} 44^{\prime} 23^{\prime \prime} \mathrm{W}\end{array}$ & & 480 \\
\hline Sampling effort & $\begin{array}{l}2440 \text { buckets. } \\
\text { days }\end{array}$ & $\begin{array}{l}3280 \text { buckets. } \\
\text { days }\end{array}$ \\
\hline
\end{tabular}

units geographically closer to PNSCo: Estação Ecológica Serra Geral do Tocantins (EESGT) (Recoder et al. 2011, Valdujo et al. 2011), Estação Ecológica de Uruçuí-Una (EEUU) (Dal Vechio et al. 2013), and Parque Nacional da Serra da Capivara (PNSCa) (Cavalcanti et al. 2014). An additional motivation in using these listings is the similar latitude in which these areas are located, forming a Cerrado-Caatinga transect. EESGT and EEUU are mostly within the Cerrado (with EEUU having some Caatinga's elements) while PNSCo and PNSCa are mostly within a Caatinga physiognomy (with PNSCo including Cerrado elements).

A dendrogram was estimated using the UPGMA clustering algorithm and Jaccard's similarity index with the program MVSP v.3.1 (Kovach 2000). Subsequently, we performed a cluster analysis using the Jaccard's coefficient, considering only the presence and absence of species and using the following local lists from different Brazilian domains: Caatinga: Planalto da Ibiapaba, Ceará (Loebmann \& Haddad 2010), Serra das Almas, Ceará (Borges-Nojosa \& Cascon 2002), Parque Nacional do Catimbau, Pernambuco (Moura et al. 2011a, b; Pedrosa et al. 2014), Ouricuri, Pernambuco (Moura et al. 2011a, b),
Fazenda Saco, Pernambuco (Moura et al. 2011a, b); Cerrado: UHE Espora, Goiás (Vaz-Silva 2007), Parque Nacional das Emas-PNE, Goiás (Nogueira et al. 2009; Valdujo et al. 2009; Kopp et al. 2010), Northern Tocantins River basin-BTN, Tocantins and Maranhão (i.e. municipalities of Estreito, Babaçulândia, Carolina and Palmeirante) (Pavan 2007), Southern Tocantins River basin-BTS, Tocantins and Goiás (i.e. municipalities of Peixe, São Salvador do Tocantins, Paranã and Minaçu) (Pavan 2007); Atlantic Forest: Mata do Buraquinho, Paraíba (Santana et al. 2008), Estação Ecológica do Tapacurá-EET, Pernambuco (Moura et al. 2011a, b); Amazon: Espigão do Oeste, Rondônia (Bernarde \& Abe 2006; Bernarde 2007; Macedo et al. 2008), Carajás, Pará (Cunha et al. 1985; Pinheiro 2010), Cacoal, Rondônia (Turci \& Bernarde 2008) Reserva Ducke, Amazonas (Lima et al. 2006; Vitt et al. 2008; Fraga et al. 2014).

\section{Results}

Sixty six species of reptiles and amphibians were found at PNSCo: 47 reptiles (21 lizards, 20 Snakes, 2 turtles and 4 amphisbaenids) and 19 amphibians (18 anurans and one caecilian) (Table 2, Figures 3, 4, 5).

Table 2. List of the herpetofauna recored at Parque Nacional Serra da Confusões (PNSCo). N: number the specimens registered; DF: Dry Forest; HAC-SGP: High Arboreal Caatinga-Serra Grande plateau; HAC-SCP: High Arboreal Caatinga-Serra das Confusões plateau; LAC: Low Arboreal Caatinga; GF: Groove Forest; HAC-LL: High Arboreal Caatinga-Lowland; "?" without information. * recorded in a recent field trip.

\begin{tabular}{|c|c|c|}
\hline & $\mathbf{N}$ & Habitat of capture \\
\hline \multicolumn{3}{|l|}{ REPTILIA } \\
\hline \multicolumn{3}{|l|}{ SQUAMATA } \\
\hline \multicolumn{3}{|l|}{ LIZARDS } \\
\hline \multicolumn{3}{|l|}{ HOPLOCERCIDAE } \\
\hline Hoplocercus spinosus Fitzinger, 1843 & 18 & $\begin{array}{l}\text { HAC-SCP, LAC, } \\
\text { HAC-LL }\end{array}$ \\
\hline \multicolumn{3}{|l|}{ IGUANIDAE } \\
\hline Iguana iguana (Linnaeus, 1758) & 2 & $?$ \\
\hline \multicolumn{3}{|l|}{ TROPIDURIDAE } \\
\hline $\begin{array}{l}\text { Stenocercus squarrosus Nogueira \& } \\
\text { Rodrigues, } 2006\end{array}$ & 13 & HAC-SGP, HAC-LL \\
\hline Tropidurus hispidus (Spix, 1825) & 84 & $\begin{array}{l}\text { HAC-SGP, HAC- } \\
\text { SCP, LAC, H AC-LL }\end{array}$ \\
\hline Tropidurus semitaeniatus (Spix, 1825) & 32 & HAC-SGP \\
\hline \multicolumn{3}{|l|}{ LEIOSAURIDAE } \\
\hline Enyalius bibronii Boulenger, 1885 & 18 & $\begin{array}{l}\text { HAC-SGP, HAC- } \\
\text { SCP, LAC, HAC-LL }\end{array}$ \\
\hline \multicolumn{3}{|l|}{ PHYLLODACTYLIDAE } \\
\hline Phyllopezus pollicaris (Spix, 1825) & 12 & HAC-SGP, LAC, GF \\
\hline \multicolumn{3}{|l|}{ SPHAERODACTYLIDAE } \\
\hline Coleodactylus brachystoma (Amaral, 1935) & 1 & LAC \\
\hline \multicolumn{3}{|l|}{ GEKKONIDAE } \\
\hline Hemidactylus brasilianus (Amaral, 1935) & 16 & $\begin{array}{c}\text { DF, HAC-SGP, } \\
\text { HAC-SCP, LAC, } \\
\text { HAC-LL }\end{array}$ \\
\hline \multicolumn{3}{|l|}{ SCINCIDAE } \\
\hline Mabuya heathi (Schmidt \& Inger, 1951) & 11 & $\begin{array}{l}\text { HAC-SGP, } \\
\text { HAC-SCP }\end{array}$ \\
\hline
\end{tabular}


Continued Table 2.

\begin{tabular}{lcc}
\hline Mabuya nigropunctata (Spix, 1825) & N & Habitat of capture \\
\hline Mabuya frenata (Cope, 1862) & 17 & $\begin{array}{c}\text { HAC-SGP, HAC- } \\
\text { SCP, LAC, HAC-LL }\end{array}$ \\
GYMNOPHTHALMAC-SGP \\
HAC-SCP
\end{tabular}

Calyptommatus confusionibus Rodrigues, Zaher \& Curcio, 2001

Colobosaura modesta (Reinhardt \& Lütken, 1862)

Micrablepharus maximiliani (Reinhardt \& Lütken, 1862)

Procellosaurinus erythrocercus Rodrigues, 1991 TEIIDAE

Ameiva ameiva (Linnaeus, 1758)

Ameivula confusioniba (Arias, De Carvalho, Rodrigues \& Zaher, 2011)

Ameivula sp.

Glaucomastix venetacauda (Arias, De

Carvalho, Rodrigues \& Zaher, 2011)

Salvator merianae (Duméril \& Bibron, 1839)

AMPHISBAENA

\section{AMPHISBAENIDAE}

Amphisbaena aff. meringoera*

Amphisbaena frontalis Vanzolini, 1991

Amphisbaena polystega (Duméril, 1851)

Amphisbaena vermicularis Wagler, 1824

SNAKES

\section{LEPTOTYPHLOPIDAE}

Trilepida cf. fuliginosa

\section{BOIDAE}

Corallus hortulanus (Linnaeus, 1758)

Epicrates assisi Machado, 1945

\section{COLUBRIDAE}

Drymarchon corais (Boie, 1827)

Drymoluber brazili (Gomes, 1918)

Leptophis ahaetulla (Linnaeus, 1758)

Oxybelis aeneus (Wagler, 1824)

Spilotes pullatus (Linnaeus, 1758)

Tantilla melanocephala (Linnaeus, 1758)

\section{DIPSADIDAE}

Apostolepis cearensis Gomes, 1915

Erythrolamprus miliaris (Linnaeus, 1758)

Erythrolamprus viridis Günther, 1862

Oxyrhopus trigeminus Duméril, Bibron \& Duméril, 1854

Philodryas nattereri Steindachner, 1870
14 HAC-SGP, HAC-LL

HAC-SGP, HAC-

45 SCP, LAC, HAC-LL

32 HAC-SGP, HAC-

32 SCP, LAC, HAC-LL

16 HAC-SGP, HAC-LL

DF, HAC-SGP,

29 HAC-SCP, LAC, HAC-LL

HAC-SGP, HACSCP, LAC, HAC-LL

23

2

LAC

HAC-SGP, HACSCP, HAC-LL

3

1

DF, HAC-SCP

$$
?
$$

LAC, GF

GF

$?$

?

HAC-SGP, LAC, GF

DF

10

HAC-SGP

$?$

$?$

$?$

HAC-SCP
HAC-SGP, LAC

Continued Table 2.

\begin{tabular}{lcc}
\hline & N & Habitat of capture \\
\hline $\begin{array}{l}\text { Philodryas olfersii (Lichtenstein, 1823) } \\
\text { Pseudoboa nigra (Duméril, Bibron \& }\end{array}$ & 1 & DF \\
$\begin{array}{l}\text { Duméril, 1854) } \\
\text { Rodriguesophis iglesiasi (Gomes, 1915) }\end{array}$ & 1 & HAC-SGP \\
Xenodon merremii (Wagler, 1824) & 1 & $?$ \\
Xenodon nattereri (Steindachner, 1867) & 1 & $?$ \\
VIPERIDAE & & $?$ \\
Crotalus durissus (Linnaeus, 1758) & 1 & $?$ \\
ARCHOSAURIA & & \\
ANAPSIDA & & \\
TESTUDINES & & \\
CHELIDAE & &
\end{tabular}

Mesoclemmys perplexa Bour \& Zaher, 2005

LAC, GF

Mesoclemmys tuberculata (Luederwaldt, 1926)

GF

\section{AMPHIBIA}

ANURAN

\section{HYLIDAE}

Corythomantis greeningi Boulenger, 1896

11

Dendropsophus soaresi (Caramaschi and Jim, 1983)

Phyllomedusa nordestina Caramaschi, 2006

Scinax gr. ruber

Scinax sp.

\section{LEPTODACTYLIDAE}

Adenomera sp. n.

Leptodactylus aff. syphax

Leptodactylus fuscus (Schneider, 1799)

Leptodactylus macrosternum MirandaRibeiro, 1926

Leptodactylus mystaceus (Spix, 1824)

Leptodactylus troglodytes Lutz, 1926

Leptodactylus vastus Lutz, 1930

Physalaemus albifrons (Spix, 1824)

Physalaemus cuvieri Fitzinger, 1826

\section{BUFONIDAE}

Rhinella granulosa (Spix, 1824)

Rhinella jimi (Stevaux, 2002)

ODONTOPHRYNIDAE

Proceratophrys cristiceps (Müller, 1883)

\section{MICROHYLIDAE}

Dermatonotus muelleri (Boettger, 1885)

GYMNOPHIONA

\section{CAECILIIDAE}

Siphonops paulensis Boettger, 1892

\section{6}

$?$

LAC

LAC

$?$

LAC, GF

LAC

LAC

$?$

LAC

HAC-SGP, LAC, HAC-LL

LAC, GF

LAC

HAC-SGP, LAC, GF

LAC, GF

GF

HAC-SGP,

LAC, GF

20 HAC-SGP, LAC 

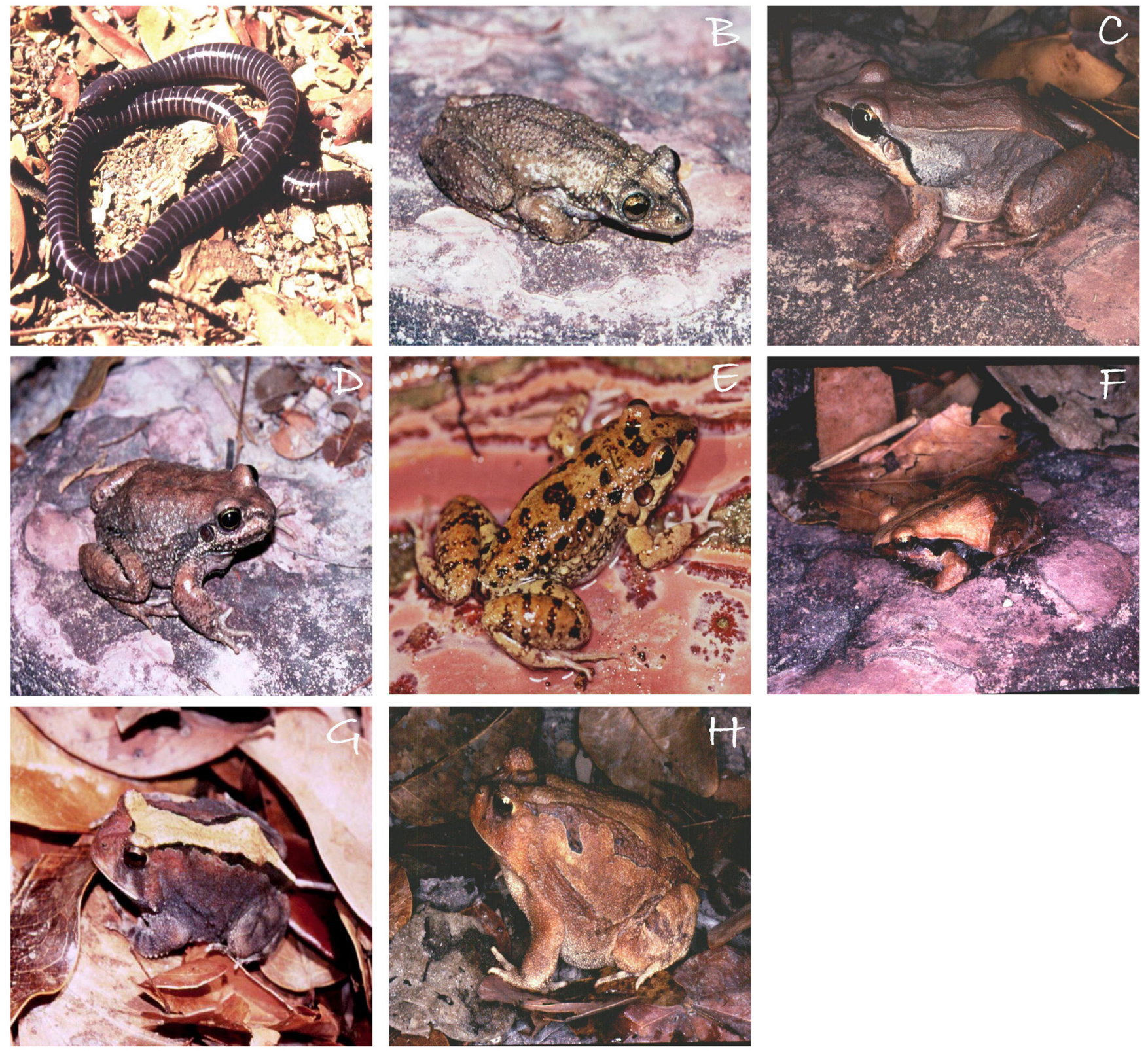

Figure 3. Some of the amphibians sampled at PNSCoe. A - Siphonops paulensis; B - Corythomantis greeningi; C - Leptodactylus mystaceus; D - Leptodactylus aff. syphax; E - Leptodactylus troglodytes; F - Physalaemus albifrons; G - Proceratophrys cristiceps (male) and H - Proceratophrys cristiceps (female).

For lizards, the rarefaction curve showed a tendency to asymptote, with decreasing standard deviation after 34 sampling days (two campaigns together) (Figure 6A), same pattern found in each campaign separately (Figure 6B). The richness estimators Chao2 and Jacknife1 recovered 19 and 20 species, respectively. For amphibians, the rarefaction curve for the two campaigns together showed only a weak tendency towards stabilization (Figure 7A), differently from the results attained for each campaign separately (Figure 7B). The richness estimators Chao2 and Jacknife1 recovered 17 and 18 species, respectively.

The complex PNSCo-PNSCa (Caatinga's units) harbors at least 74 species, and share 30 species, as follows: 52 reptiles (24 lizards, 4 amphisbaenas, 22 snakes and 2 turtles) and 22 amphibians (20 anurans and two caecilians). Adding the closer EEUU and EESGT Cerrado's units, the number of species increases to 191 (about 12.5\% of Brazilian species): 130 reptiles (45 lizards, 10 amphisbaenas, 69 snakes, 4 turtles and 2 crocodilians) and 61 amphibians (59 anurans and two caecilians) (Table 3).

Sixteen species occured in all four units while 17 species were shared among three of them, 32 species were shared only between EEUU and EESGT, 8 species between PNSCo and PNSCa, 5 species between PNSCo and EEUU, and 4 between PNSCo and EESGT. Only one species was shared between PNSCa and EEUU and none were exclusive of PNSCa and EESGT together. Seven species occurred exclusively in PNSCa, 17 in PNSCo, 20 in EEUU, and 64 in EESGT (Table 3). 

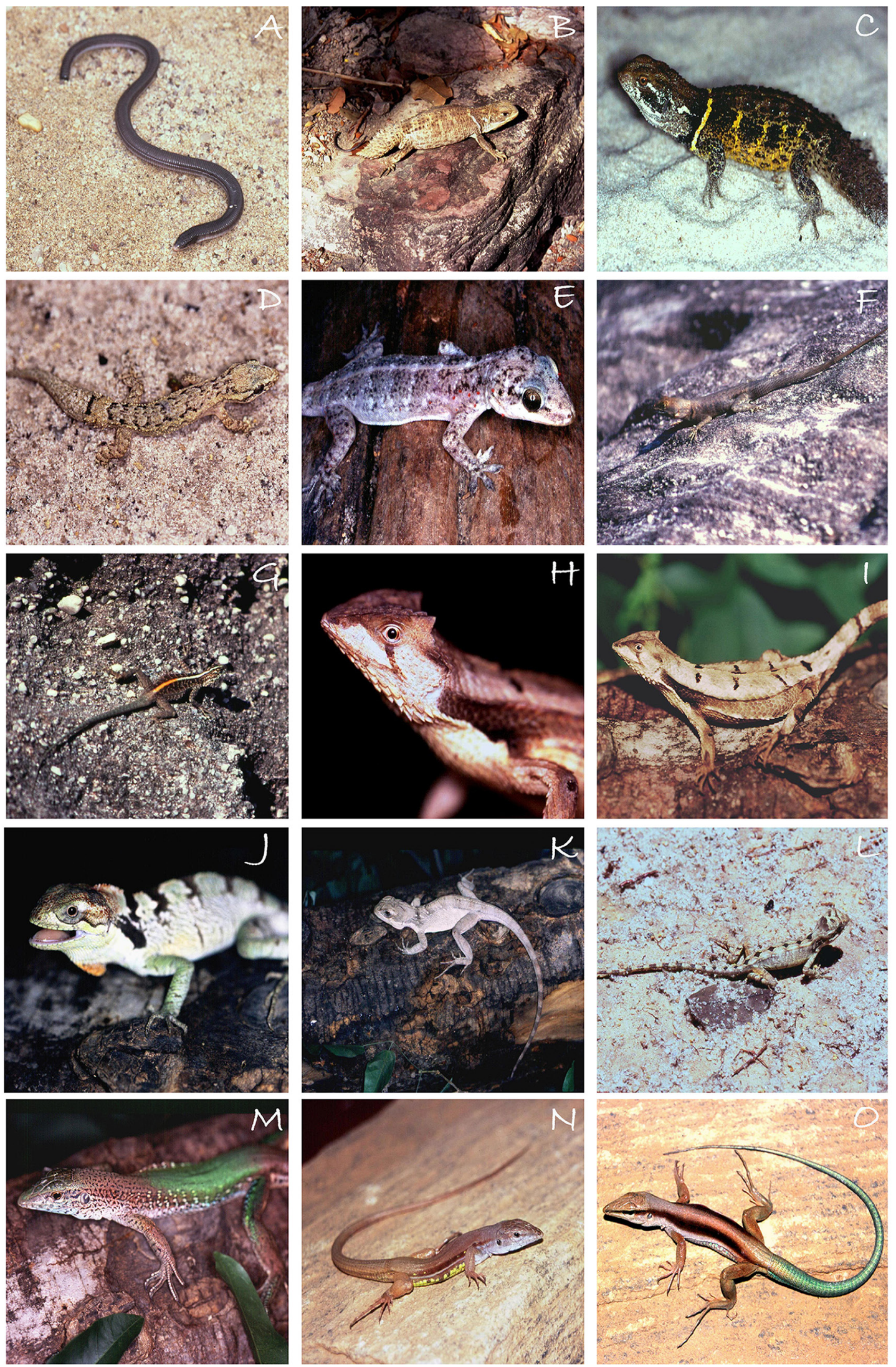

Figure 4. Some of the reptile sampled at PNSCo. A - Amphisbaena vermicularis; B - Hoplocercus spinosus (female); C - Hoplocercus spinosus (male); D - Hemidactylus brasilianus; E - Phyllopezus pollicaris; F - Tropidurus hispidus; G - Tropidurus semitaeniatus; $\mathrm{H}$ and I - Stenocercus squarrosus; J - Enyalius bibronii (male); K - Enyalius bibronii (female); L - Enyalius bibronii (juvenile); M - Ameiva ameiva; N - Ameivula confusioniba; O - Glaucomastix venetacauda. 

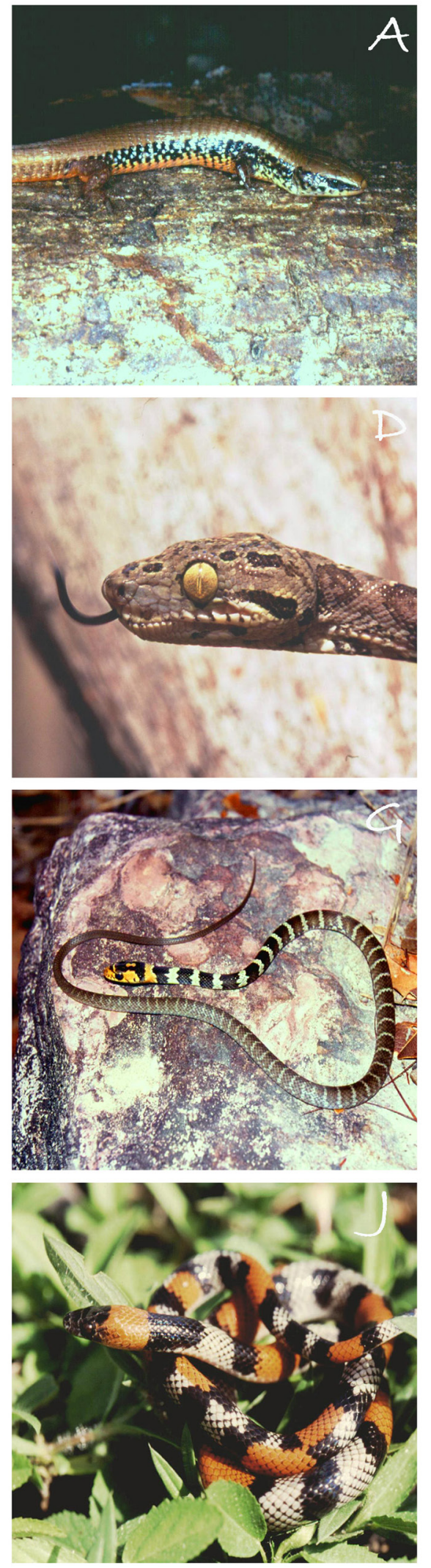
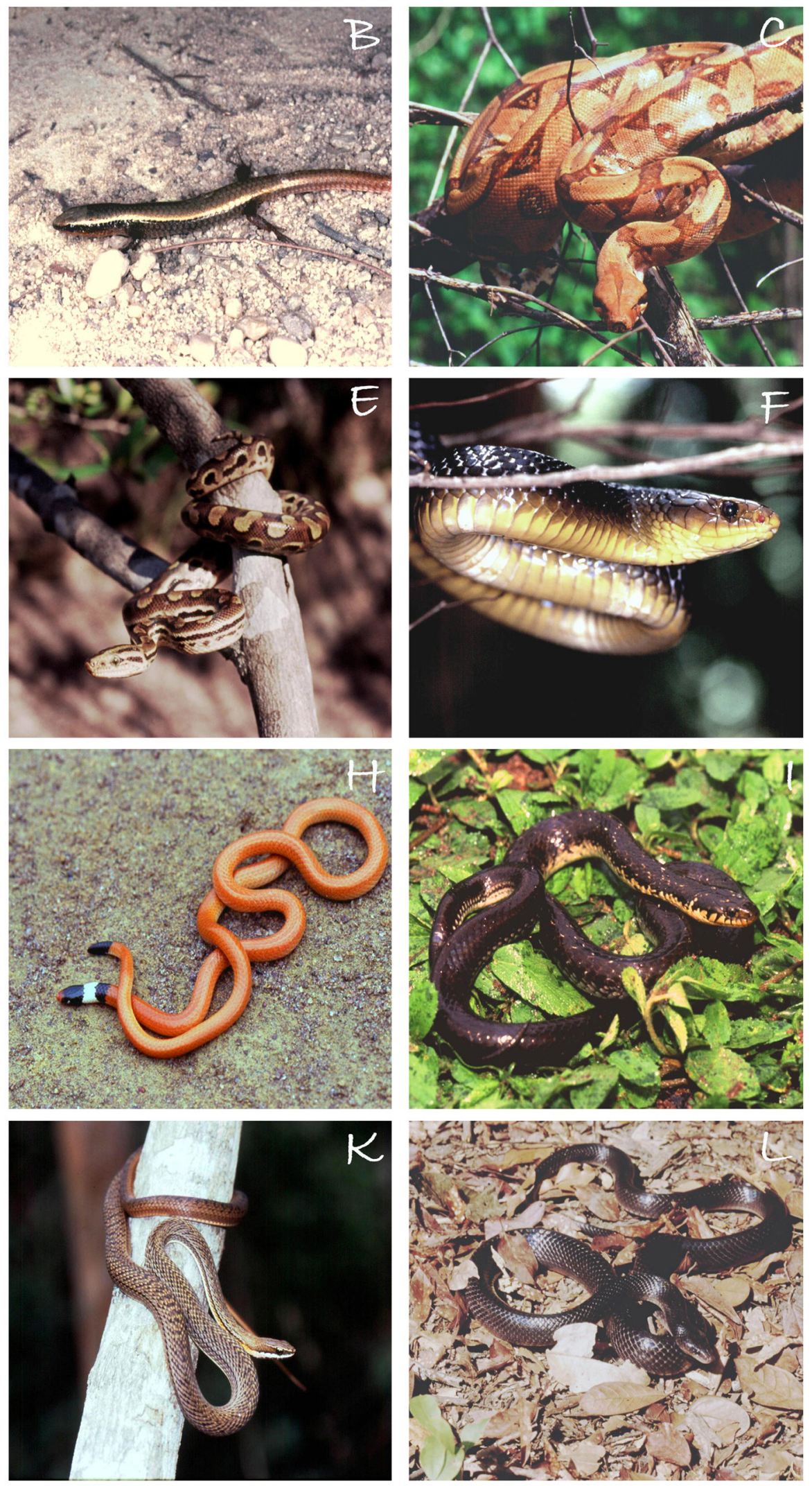

Figure 5. Some of the reptile sampled at PNSCo. A - Colobosaura modesta; B - Procellosaurinus erythrocercus; C - Boa constrictor; D - Corallus hortulanus; E - Epicrates assisi; F - Drymarchon corais; G - Drymoluber brazili; H - Apostolepis cearensis; I - Erythrolamprus miliaris; J - Oxyrhopus trigeminus; K - Philodryas nattereri; L - Pseudoboa nigra. 
Lizards
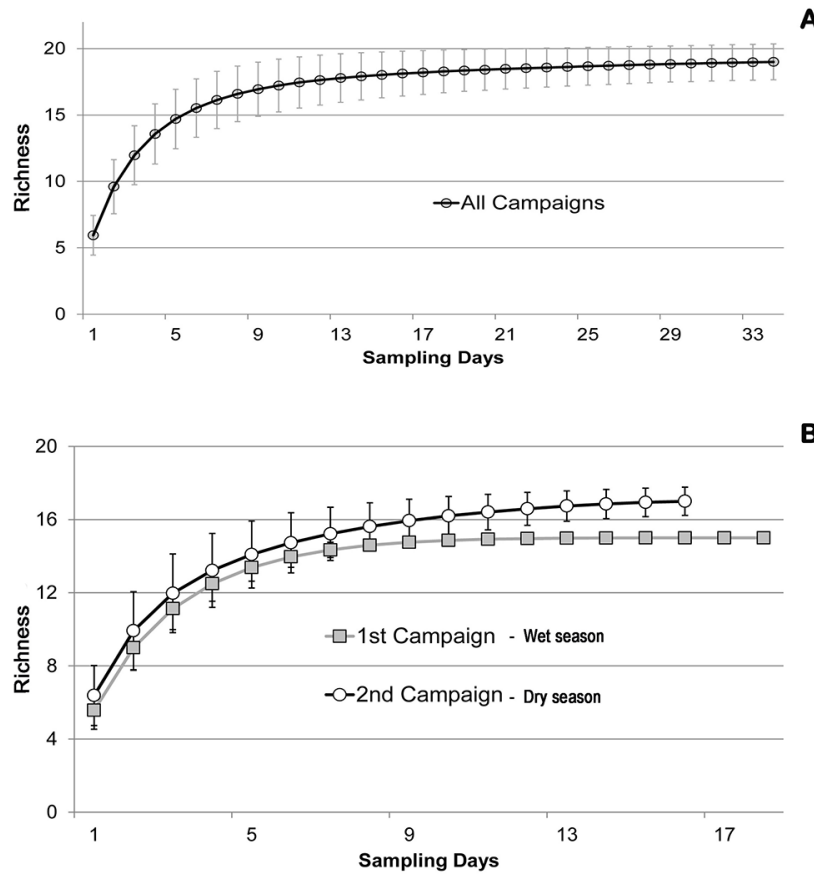

Figure 6. Rarefaction curve for lizards. A - after 34 sampling days (two campaign together); B - for each campaign separately.
A

B
Amphibians
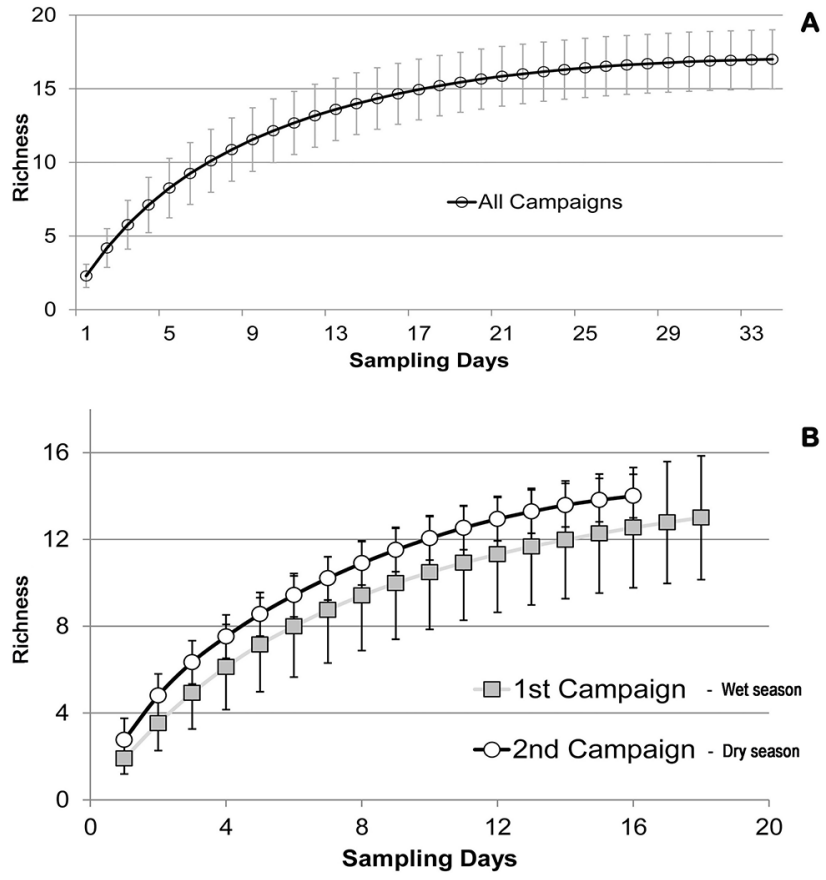

Figure 7. Rarefaction curve for amphibians. A - after 34 sampling days (two campaign together); B - for each campaign separately.
B

Table 3. Regional Herpetofaunal list, with presence "X” or "-“" absence of species for each conservation unit. PNSCo: Parque Nacional da Serra das Confusões (present work); EEUU: Estação Ecológica de Uruçuí-Una (Dal Vechio et al. 2013); EESGT: Estação Ecológica Serra Geral do Tocantins (Recoder et al. 2011, Valdujo et al. 2011); PNSCa: Parque Nacional da Serra da Capivara (Cavalcanti et al. 2014).

\begin{tabular}{cccc} 
PNSCa & $\begin{array}{c}\text { PNSCo } \\
\text { (Caatinga } \\
\text { with Cerrado } \\
\text { elements) }\end{array}$ & $\begin{array}{c}\text { EEUU (Cerrado } \\
\text { with Caatinga } \\
\text { elements) }\end{array}$ & $\begin{array}{c}\text { EESGT } \\
\text { (Cerrado) }\end{array}$ \\
\hline & & &
\end{tabular}

\section{REPTILIA}

\section{SQUAMATA}

\section{LAGARTOS}

\section{HOPLOCERCIDAE}

Hoplocercus spinosus Fitzinger, 1843

\section{IGUANIDAE}

Iguana iguana (Linnaeus, 1758)

\section{TROPIDURIDAE}

Stenocercus squarrosus Nogueira \& Rodrigues, 2006

Stenocercus quinarius Nogueira \& Rodrigues, 2006

Tropidurus cf. oreadicus

Tropidurus helenae (Manzani \& Abe, 1990)

Tropidurus oreadicus Rodrigues, 1987

Tropidurus semitaeniatus (Spix, 1825)

Tropidurus hispidus (Spix, 1825)

\section{LEIOSAURIDAE}

Enyalius bibronii Boulenger, 1885

\section{POLYCHROTIDAE}

Norops chrysolepis Duméril \& Bibron, 1837

Norops brasiliensis Vanzolini \& Williams 1970

Norops meridionalis Boettger, 1885

$\begin{array}{cc}- & X \\ X & X \\ X & X \\ - & - \\ - & - \\ X & - \\ - & X \\ X & X \\ X & \end{array}$

$\mathrm{X}$

$\mathrm{X}$

\section{.}




\begin{tabular}{|c|c|c|c|c|}
\hline & $\begin{array}{c}\text { PNSCa } \\
\text { (Caatinga) }\end{array}$ & $\begin{array}{c}\text { PNSCo } \\
\text { (Caatinga } \\
\text { with Cerrado } \\
\text { elements) }\end{array}$ & $\begin{array}{c}\text { EEUU (Cerrado } \\
\text { with Caatinga } \\
\text { elements) }\end{array}$ & $\begin{array}{c}\text { EESGT } \\
\text { (Cerrado) }\end{array}$ \\
\hline \multicolumn{5}{|l|}{ PHYLLODACTYLIDAE } \\
\hline Gymnodactylus amarali Barbour, 1925 & - & - & - & $\mathrm{X}$ \\
\hline \multicolumn{5}{|l|}{ SPHAERODACTYLIDAE } \\
\hline Coleodactylus brachystoma (Amaral, 1935) & - & $\mathrm{X}$ & $\mathrm{X}$ & $\mathrm{X}$ \\
\hline Coleodactylus cf. meridionalis & - & - & - & $\mathrm{X}$ \\
\hline \multicolumn{5}{|l|}{ GEKKONIDAE } \\
\hline Hemidactylus brasilianus (Amaral, 1935) & $\mathrm{X}$ & $\mathrm{X}$ & $\mathrm{X}$ & $\mathrm{X}$ \\
\hline Mabuya cf. heathi & - & - & - & $\mathrm{X}$ \\
\hline Mabuya heathi (Schmidt \& Inger, 1951) & - & $\mathrm{X}$ & - & - \\
\hline Mabuya cf. nigropunctata & - & - & - & $\mathrm{X}$ \\
\hline Mabuya nigropunctata (Spix, 1825) & $\mathrm{X}$ & $\mathrm{X}$ & $\mathrm{X}$ & - \\
\hline Mabuya frenata (Cope, 1862) & - & $\mathrm{X}$ & - & $\mathrm{X}$ \\
\hline \multicolumn{5}{|l|}{ GYMNOPHTHALMIDAE } \\
\hline Bachia oxyrhina Rodrigues et al., 2008 & - & - & - & $\mathrm{X}$ \\
\hline Calyptommatus confusionibus Rodrigues, Zaher \& Curcio, 2001 & - & $\mathrm{X}$ & - & - \\
\hline Cercosaura ocellata Wagler, 1830 & - & - & - & $\mathrm{X}$ \\
\hline Ameiva ameiva (Linnaeus, 1758) & $\mathrm{X}$ & $\mathrm{X}$ & $\mathrm{X}$ & $\mathrm{X}$ \\
\hline Ameivula confusioniba (Arias, De Carvalho, Rodrigues \& Zaher, 2011) & - & $\mathrm{X}$ & - & - \\
\hline Ameivula cf. mumbuca & - & - & $\mathrm{X}$ & - \\
\hline Ameivula jalapensis (Colli et al., 2009) & - & - & - & $\mathrm{X}$ \\
\hline Ameivula mumbuca (Colli et al., 2003) & - & - & - & $\mathrm{X}$ \\
\hline Ameivula ocellifera (Spix, 1825) & $\mathrm{X}$ & - & - & - \\
\hline Ameivula sp. & - & $\mathrm{X}$ & - & - \\
\hline Glaucomastix venetacauda (Arias, De Carvalho, Rodrigues \& Zaher, 2011) & $\mathrm{X}$ & $\mathrm{X}$ & - & - \\
\hline Kentropyx aff. paulensis Boettger, 1893 & - & - & - & $\mathrm{X}$ \\
\hline Kentropyx calcarata Spix, 1825 & - & - & $\mathrm{X}$ & - \\
\hline Salvator duseni (Lönnberg, 1910) & - & - & - & $\mathrm{X}$ \\
\hline Salvator merianae (Duméril \& Bibron, 1839) & $\mathrm{X}$ & $\mathrm{X}$ & $X$ & $\mathrm{X}$ \\
\hline Tupinambis quadrilineatus Manzani \& Abe, 1997 & - & - & $\mathrm{X}$ & $\mathrm{X}$ \\
\hline \multicolumn{5}{|l|}{ AMPHISBAENAS } \\
\hline \multicolumn{5}{|l|}{ AMPHISBAENIDAE } \\
\hline Amphisbaena acrobeles (Ribeiro, Castro-Mello \& Nogueira, 2009) & - & - & - & $\mathrm{X}$ \\
\hline Amphisbaena aff. miringoera* & $\mathrm{X}$ & $\mathrm{X}$ & $\mathrm{X}$ & $\mathrm{X}$ \\
\hline
\end{tabular}




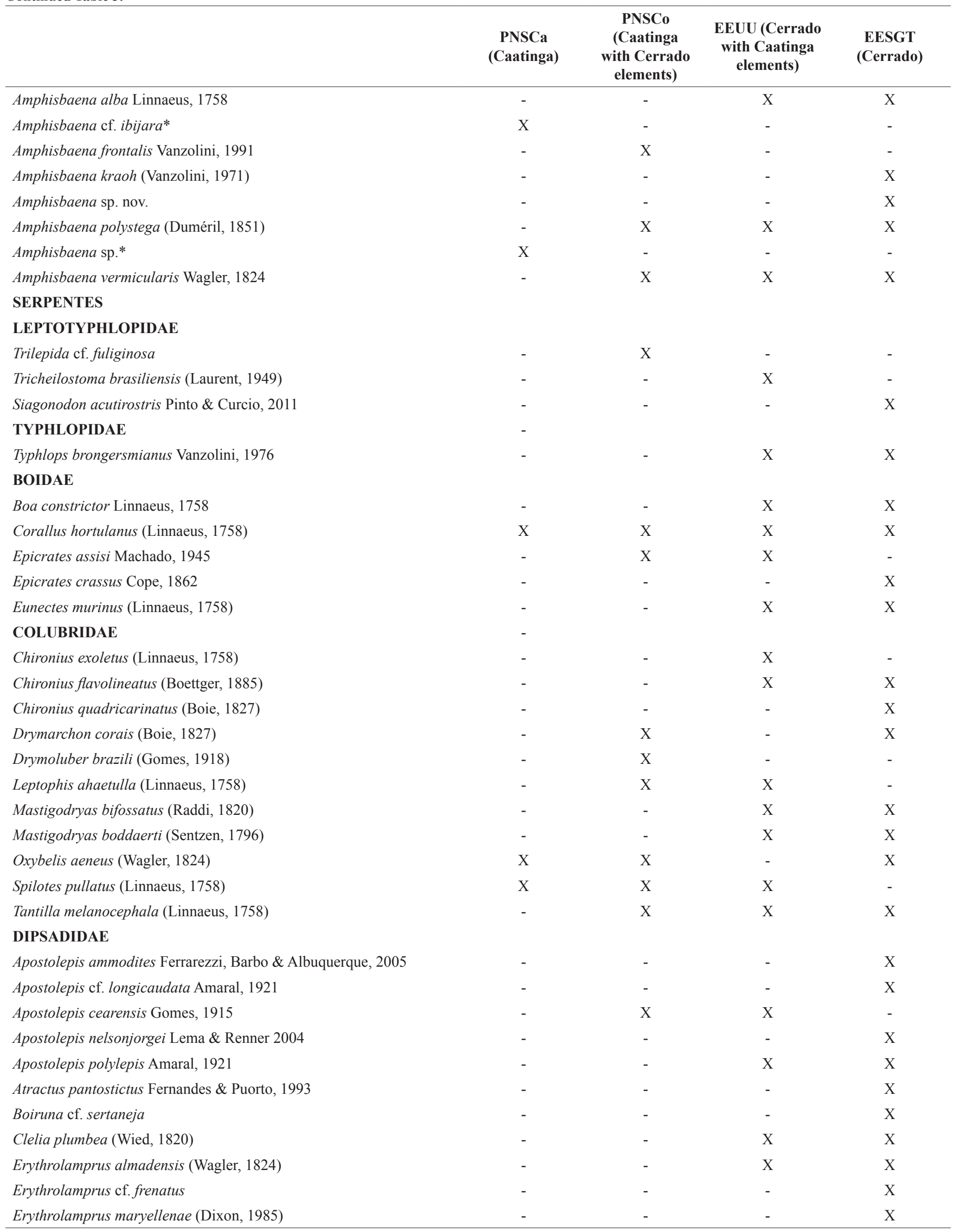




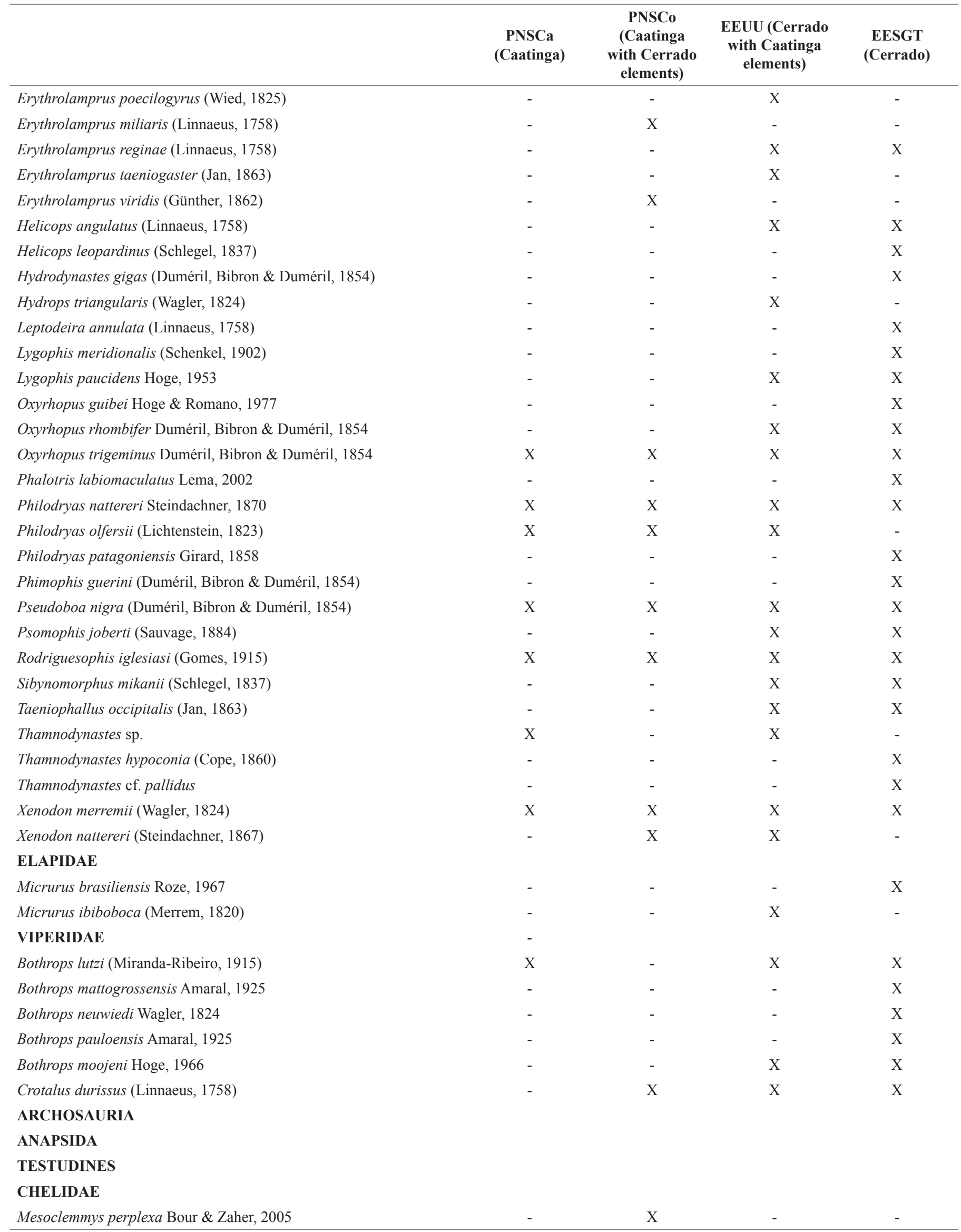




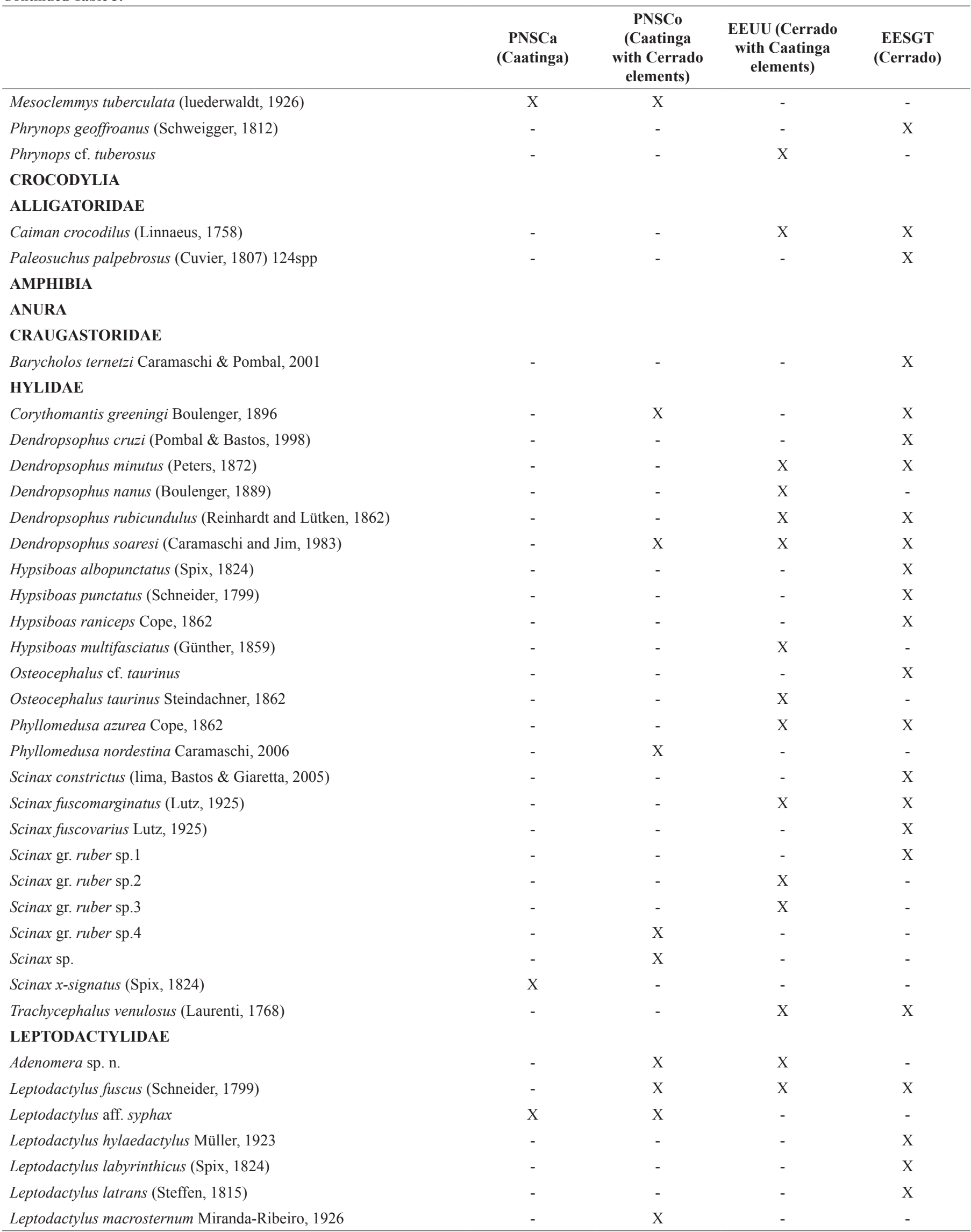




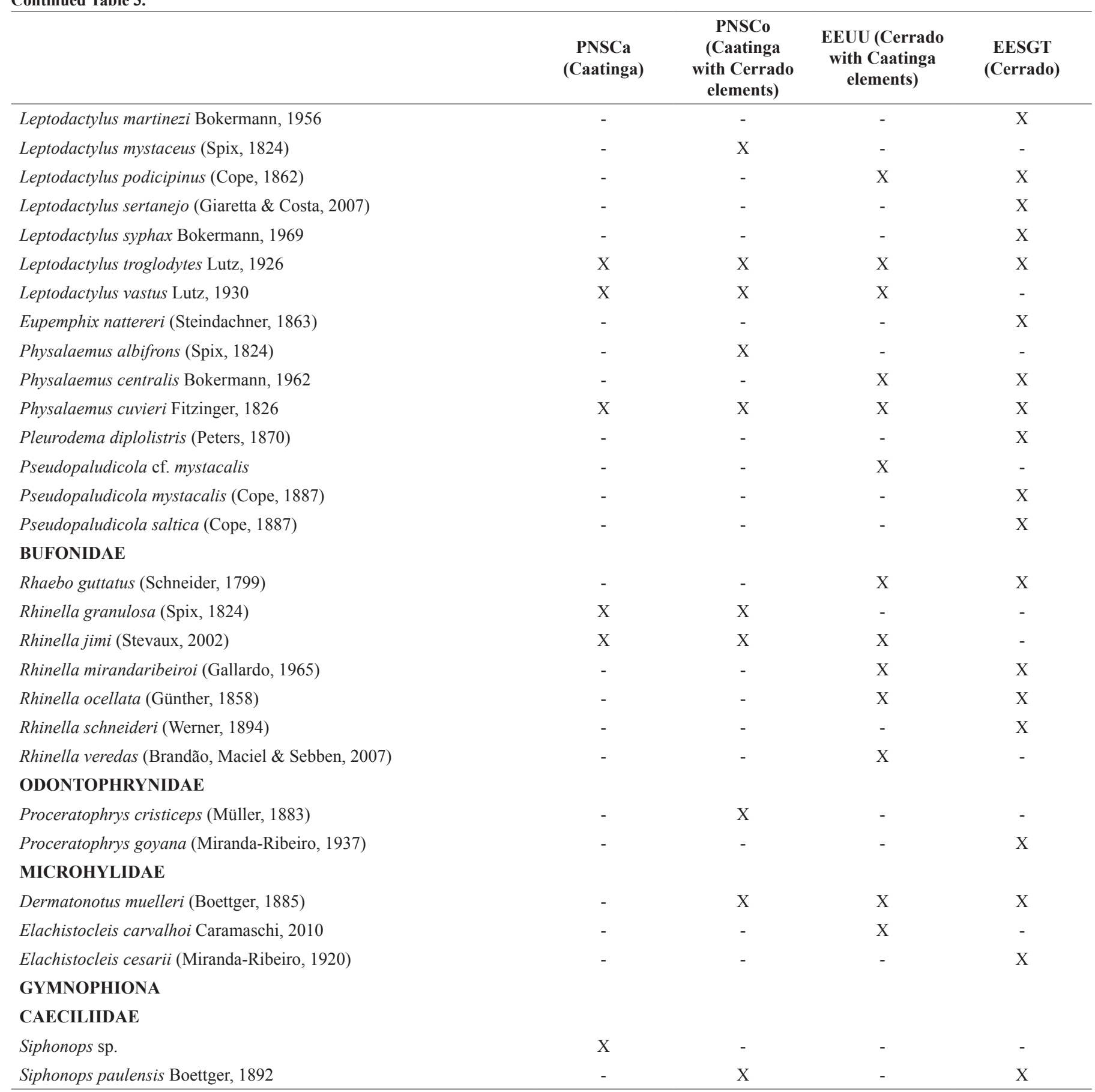

The cluster analysis recovered PNSCo and PNSCa within the Caatinga group, and EEUU and EESGT within the Cerrado group, indicating a strong species turnover in the contact region between Caatinga and Cerrado in southern Piauí (Figure 8). The same pattern was recovered for lizards and amphibians when analyzed separately (Data not shown).

\section{Discussion}

The herpetofauna of PNSCo is one of the most diverse of the Caatinga biome, except for the fauna from the isolated forest patches of northeastern Brazil, usually referred as "Brejos Nordestinos". A total of 66 species were recorded (74 species when taking together PNSCo and PNSCa), equating in diversity with previously well sampled areas like Exu in Pernambuco, Valença in Piauí, and Xingó in Alagoas (with 53, 42 and 41 species, respectively) (Rodrigues, 2003a).

Part of the high diversity found in the PNSCo and PNSCa taken together can be explained by landscape heterogeneity, which, in turn, also characterizes the Cerrado units and strongly differs from Caatinga (sensu stricto) areas where the landscape physiognomy is far more homogeneus. However, since the PNSCo and PNSCa are situated in an ecotonal area between these two biomes, they harbor a mix of 


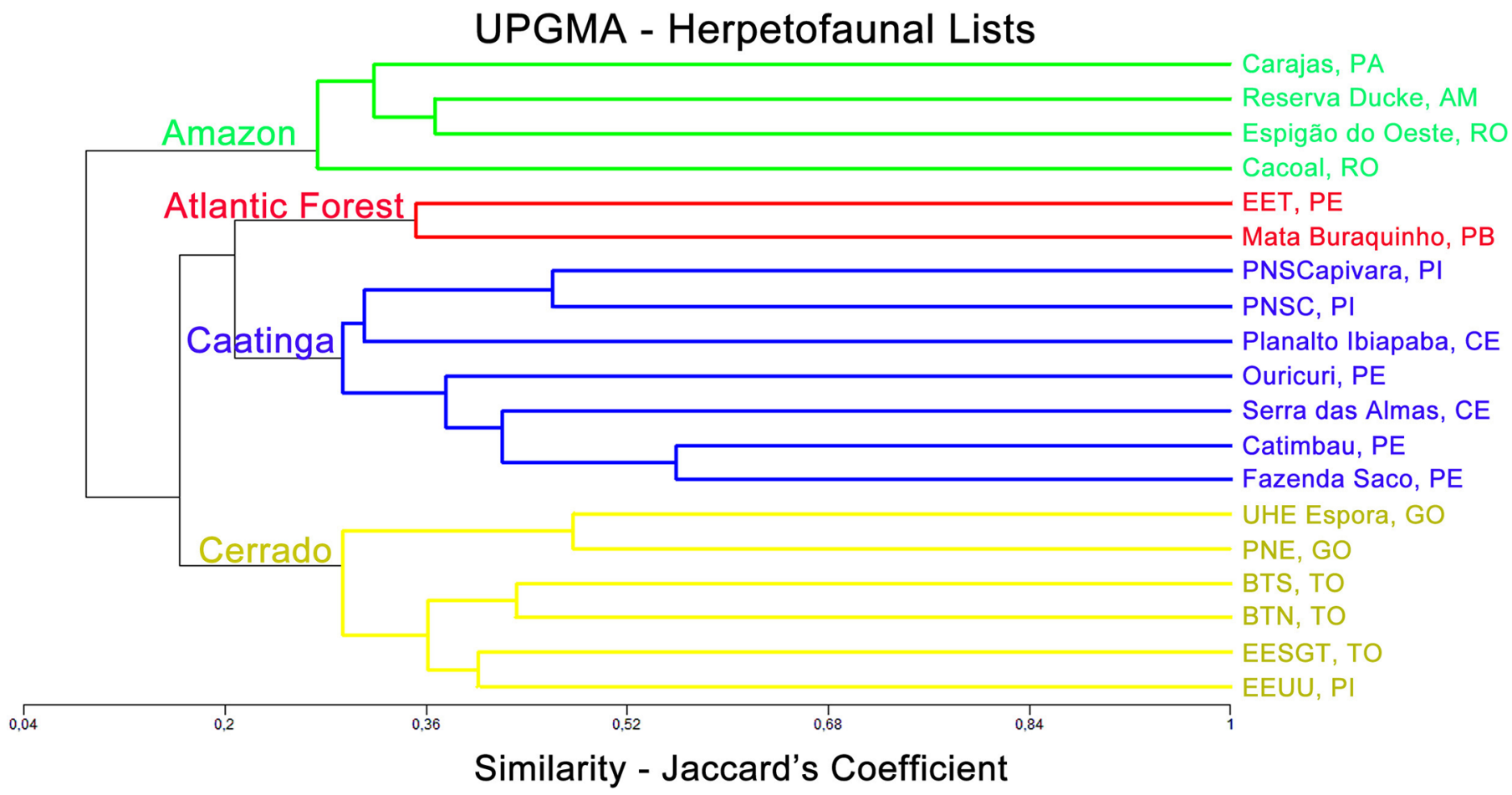

Figure 8. UPGMA of herpetofaunal lists from different Brazilian biomes. Green: Amazon; Red: northwest Atlantic Forest; Blue: Caatinga and Yellow: Cerrado.

Caatinga and Cerrado elements, forming an heterogeneous landscape. This results in high species diversity, with species typical from Cerrado (e.g. Hoplocercus spinosus, Coleodactylus brachystoma, Colobosaura modesta) and Caatinga (e.g. Epicrates assisi, Dermatonotus mulleri, Physalaemus albifrons, Corythomantis greeningi, Rhinella jimi, Proceratophrys cristiceps) coexisting in the area. Adding to phytogeomorphological features, historical events also could be acting to increase the local diversity in the southwestern Piauí, as the historical contacts now lost between sandy soils from this region and those from the São Francisco dunes (Rodrigues et al. 2001).

The observed richness of lizards and amphibians might be close to the real diversity in PNSCo since species rarefaction curves for both groups tend toward an asymptote after 34 sampling days (stronger for lizards) and richness estimators recover close values to those obtained. However, some new species records are expected in the future since arboreal, aquatic, fossorial species and ones with explosive reproduction are always more difficult to sample and tend to be underestimated even in long-term and/or large-scale inventories.

Indeed, the most diverse lists of local herpetofauna are those based on intensive field work with long periods of sampling along different climatic seasons and/or mega infrastructure projects with extensive devastation of natural habitats, such as hydroelectric power plants (Pavan \& Dixo 2004, Vaz-Silva et al. 2007, Silva Junior et al. 2005, Loebmann \& Haddad 2010, Nogueira et al. 2010, Silveira et al. 2010, Recoder et al. 2011, Valdujo et al. 2011, Dal Vechio et al. 2013, present work). In addition, long-term surveys provide necessary material to understand the basic biology of the species (Vitt 1982; Cruz 1994; Mesquita \& Colli 2003; Werneck et al. 2009; Dal Vechio et al. 2014, 2015).
Sampling efforts at PNSCo resulted in the discovery and description of five new species: Calyptommatus confusionibus (Rodrigues et al. 2001), Mesoclemmys perplexa (Bour \& Zaher 2005), Stenocercus squarrosus (Nogueira \& Rodrigues 2006), Ameivula confusioniba and Glaucomastix venetacauda (Arias et al. 2011a). Another three are candidates to new species. Adenomera sp., was detected as a possible new species in a recent molecular study (Fouquet et al. 2014). This species also occurs at EEUU, being probably distributed along the northern Cerrado and ecotonal areas between this domain and the neighbors. Leptodactylus aff. syphax has recently been recognized as an undescribed new species (Loebmann \& Haddad 2010; Andrade et al. 2011; Ribeiro et al. 2012; Cavalcanti et al. 2014). Ameivula sp. seems to be a hybrid between $A$. confusioniba and G. venetacauda (Arias pers. comun).

Five additional species are worthy of comment. The five specimens of Trilepida sampled in the PNSCo have scale counts that are intermediate between T. koppesi and T. fuliginosa. Although quantitatively more similar to T. koppesi, they present qualitative characters that resemble T. fuliginosa (Passos et al. 2006). Trilepida koppesi is distributed throughout the southern Cerrado region (São Paulo, eastern Mato Grosso do Sul and southern Goiás states), while T. fuliginosa is distributed in the northern part of the Cerrado, with the nearest record in Palmas (Tocantins basin), about $520 \mathrm{~km}$ in straight line far from PNSCo (Passos et al. 2006). Sampled specimens in PNSCo could either be an undescribed species or represent a large distribution extension for Trilepida fuliginosa and the first record of the species for the Caatinga domain.

Other interesting species is Siphonops paulensis, which is widely distributed in Brazil, with only a few records for the Caatinga biome 
(Taylor 1968, 1970; Santana et al. 2015). The specimen of S. paulensis represents the first record for the state of Piauí and fills an important distribution gap for the group in northwestern Brazil. However, it is likely that $S$. paulensis represents a complex of cryptic species (Wilkinson, pers. comm.). Indeed, Loebmann and Haddad (2010) already pointed out the complex taxonomic nature of this species when they registered Siphonops for the "Brejo Nordestino" Planalto de Ibiapaba and considered their record as possibly representing an undescribed species related to $S$. paulensis. Here we treat the population in PNSCo as $S$. paulensis, pending further studies that could clarify the taxonomic status of populations considered to belong to this widely distributed species.

A recent phylogenic work of Tropidurus semitaeniatus showed the complexity of the the species, with several lineages candidates to fully species, inclusive, there are two distinct of these lineages that occur in PNSCo, being one of them exclusive to the park; and there is another exclusive lineage to EEUU (Werneck et al. 2015). Here, we maintain T. semitaeniatus as just one species until further taxonomic approach.

Amphisbaena frontalis is until now known only from the type locality at the left bank of middle São Francisco River in Alagoado, Bahia state. The record in PNSCo expands its distribution on approximately 270 $\mathrm{km}$ northwest of the type locality (Vanzolini 1991); and highlights an historical event of sand contact with southwestern Piauí and São Francisco Dunes as mentioned above. Finally, Hoplocercus spinosus is a typical Cerrado species that also occurs in contact areas with forested biomes. In PNSCo, H. spinosus was found in a forested Caatinga, representing the easternmost record and first for the species within the Caatinga domain (Torres-Carvajal et al. 2011).

The PNSCo together with other regional conservation units of similar latitude (i.e. EESGT, EEUU and PNSCa) protect an area of ca. $200.000 \mathrm{Km}^{2}$ in northern Cerrado and western Caatinga. There is a widespread herpetofaunal species throughout this landscape, occuring in all these four units, however, the fauna is more similar between Cerrado's units (EESGT + EEUU) and Caatinga's ones (PNSCo and PNSCa) but not so much between them. Despite the geographical proximity between these Cerrado and Caatinga parks, there is a turnover of congeneric species that seems to be related to the shift of domains (e.g. Tropirurus oreadicus X Tropirurus hispidus; Stenocercus quinarius X Stenocercus squarrosus; Ameivula mumbuca X Ameivula confusioniba; Procellosaurinus erythrocercus X Vanzosaura savanicola; Epicrates crassus X Epicrates assisi; Phyllomedusa azurea $\mathrm{X}$ Phyllomedusa nordestina; Leptodactylus syphax X Leptodactylus aff. syphax; Physalaemus centralis X Physalaemus albifrons; Rhinella mirandaribeiroi X Rhinella granulosa; Rhinella schneideri $\mathrm{X}$ Rhinella jimi; Proceratophrys goyana X Proceratophrys cristiceps). This shift on is also recovered in the similarity analysis, with PNSCo and PNSCa included within the "Caatinga" group while EESGT and EEUU units cluster within the "Cerrado" group, indicating that the units harbor a typical faunal of each biome.

Our results show that the PNSCo harbors one of the most diverse herpetofauna among the inventoried localities within the Caatinga domain, conferring to the park a strategic role for the conservation of the remaining regions of this vanishing domain. Our results also indicate that, despite geographical proximity, the northeastern Cerrado and Caatinga units studied still retain high levels of diversity and uniqueness with low faunal similarities between domains, evidencing a high species turnover.

\section{Acknowledgements}

We are indebted to Deocleciano Guedes Ferreira for his support as head of the IBAMA Regional Office of Piauí. We would like to thank the participation in the field work of the colleagues Alexandre R. Percequillo, Felipe F. Curcio, Giovanna G. Montingelli, Luis F. Silveira, Marcos Pérsio Dantas Santos, Paulo Balduino, and Pedro Nunes. This research was supported by grants to HZ from Fundação de Amparo Pesquisa do Estado de São Paulo (BIOTA/FAPESP grant number 2011/50206-9) and Conselho Nacional de Desenvolvimento Científico e Tecnológico (CNPq grant numbers 565046/2010-1 and 303545/2010-0).

\section{References}

ANDRADE, E.B.; GUIMARÃES, R.; LEITE Jr., J.M.A. \& LEITE, J.R.S.A. 2011. Amphibia, Anura, Leptodactylidae, Leptodactylus syphax Bokermann, 1969: Distribution extension and geographic distribution map. CheckList, 7(5):592-593

ARIAS, F.; CARVALHO, C.M.; RODRIGUES, M.T. \& ZAHER, H. 2011a. Two new species of Cnemidophorus (Squamata: Teiidae) from the Caatinga, northwest Brazil. Zootaxa, 2787:37-54.

ARIAS, F.; CARVALHO, C.M., RODRIGUES, M.T. \& ZAHER, H. 2011b. Two new species of Cnemidophorus (Squamata: Teiidae) of the C. ocellifer group, from Bahia, Brazil. Zootaxa, 3022: 1-21.

ARIAS, F.J., TEIXEIRA Jr., M., DE CARVALHO, C.M., RECODER, R., ZAHER, H. and RODRIGUES, M.T. 2014. Whiptail lizards in South America: a new Ameivula (Squamata, Teiidae) from Planalto dos Gerais, Eastern Brazilian Cerrado. Amphibia-Reptilia, 35: 227-242.

BERNARDE, P.S. \& ABE, A.S. 2006. A snake community at Espigão do Oeste, Rondônia, Southwestern Amazon, Brazil. South American Journal of Herpetology, 1:102-113.

BERNARDE, P.S. 2007. Ambientes e temporada de vocalização da anurofauna no Município de Espigão do Oeste, Rondônia, Sudoeste da Amazônia - Brasil (Amphibia: Anura). Biota Neotropica, 7:87-92.

BORGES-NOJOSA, D.M. \& CASCON, P. 2005. Herpetofauna da Área Reserva da Serra das Almas, Ceará. In: Araújo, F.S.; Rodal, M.N.J. \& Barbosa, M.R.V. (Eds.), Análise das Variações da Biodiversidade do Bioma Caatinga. Ministério do Meio Ambiente, Brasília, p.245-260.

BOUR, R. \& ZAHER, H. 2005. A new species of Mesoclemmys, from the open formations of northeastern Brazil (Chelonii, Chelidae). Papéis Avulsos de Zoologia, 45(24): 295-311.

BROOKS, D.R.; MAYDEN, R.L. \& MCLENNAN, D.A. 1992. Phylogeny and biodiversity: conserving our evolutionary legacy. Trends in Ecology and Evolution, 7:55-59.

CASTRO, A.A.J.F. 2000. Cerrado do Brasil e do Nordeste. In: Benjamin, A. H. \& Sícoli, J. C.M. (Eds). Agricultura e meio ambiente (Agriculture and the environment). São Paulo. IMESP, p.79-87.

CAVALCANTI, L.B.Q.; COSTA, T.B.; COLLI, G.R.; COSTA, G.C.; FRANÇA, F.G.R.; MESQUITA, D.O.; PALMEIRAS, C.N.S.; PELEGRIN, N.; SOARES, A.H.B.; TUCKER, D.B. \& GARDA, A.A. 2014. Herpetofauna of protected areas in the Caatinga II: Serra da Capivara National Park, Piauí, Brazil. CheckList, 10(1)18-27.

CAVALCANTI, R. \& JOLY, C.A. 2002. Biodiversity and conservation priorities in the Cerrado region. In: Oliveira P.S. \& Marquis, R.J. (Eds), The Cerrados of Brazil: Ecology and Natural History of a Neotropical Savanna. Columbia University Press, New York, p.351-367. 
COLLI, G.R.; BASTOS, R.P. \& ARAÚJO, A.F.B. 2002. The character and dynamics of the Cerrado Herpetofauna. In: Oliveira P.S. \& Marquis, R.J. (Eds), The Cerrados of Brazil: Ecology and Natural History of a Neotropical Savanna. Columbia University Press, New York, p.223-241.

COLLI, G.R.; CALDWELL, J.P.; COSTA, G.C.; GAINBURY, A.M.; GARDA, A.A.; MESQUITA, D.O.; FILHO, C.M.; SOARES, A.H.B.; SILVA, V.N.; VALDUJO, P.H.; VIEIRA, G.H.C.; VITT, L.J.; WERNECK, F.P.; WIEDERHECKER, H.C. \& ZATZ, M.G. 2003. A new species of Cnemidophorus (Squamata, Teiidae) from the Cerrado Biome in central Brazil. Occasional Papers of the Sam Noble Oklahoma Museum of Natural History, 14:1-14.

COLWELL, R.K. 2006. EstimateS: Statistical estimation of species richness and shared species from samples. Version 8.0. Available at: http://purl. oclc.org/estimates.

CRUZ, F.B. 1994. Actividad reprodutiva en Vanzosaura rubricauda (Sauria: Teiidae) del chaco accidental en Argentina. Cuaderno de Herpetología, 8: $112-1118$.

CUNHA, O.R.; NASCIMENTO, F.P. \& AVILA-PIRES, T.C.S. 1985. Os répteis da área de Carajás, Pará, Brasil (testudines e Squamatas). Publicações Avulsas do Museu Paraense Emílio Goeldi, 40:9-92.

DAL VECHIO, F.; RECODER, R.S.; HUSSAN, Z. \& RODRIGUES, MT. 2013. The herpetofauna of the Estação Ecológica de Uruçuí-Una, state of Piauí, Brazil. Papéis Avulsos de Zoologia, 53: 225- 243.

DAL VECHIO, F.; RECODER, R.S.; HUSSAN, Z. \& RODRIGUES, MT 2014. Natural history of Micrablepharus maximiliani (Squamata, Gymnophthalmidae) in a Cerrado region of northeastern Brazil. Zoologia, 31(2): 114-118

DAL VECHIO, F., TEIXEIRA Jr., M. MOLLO-NETO, A. and RODRIGUES, M.T. 2015. On the snake Siphlophis worontzowi (Prado, 1940) notes on its distribution, diet and morphological data. Checklist, 11(1): 1534.

EITEN, G. 1972. The cerrado vegetation of Brazil. Revista Brasileira de Botânica, 38:201-341.

FERRAREZZI, H.; BARBO, F.E. \& ALBURQUERQUE, C.E. 2005. Phylogenetic relationships of a new species of Apostolepis from Brazilian Cerrado with notes on the assimilis group (Serpentes: Colubridae: Xenodontinae: Elapomorphini). Papéis Avulsos de Zoologia, 45:215-229.

FOUQUET, A.; CASSINI, C.S.; BAPTISTA, C.F.; PECH, N. \& RODRIGUES, M.T. 2014. Species delimitation, patterns of diversification and historical biogeography of the Neotropical frog genus Adenomera (Anura, Leptodactylidae). Journal of Biogeography, 41(5): 855-870.

FRAGA, R.; LIMA, A.P.; PRUDENTE, A.L.C. \& MAGNUSSON, W.E. 2013. Guide to the snakes of the Manaus region, central Amazonia. Editora INPA, Manaus, Brasil, Pp. 303.

FRANÇA, F.G.R. \& ARAÚJO, A.F.B. 2006. The conservation status of snakes in Central Brazil. South American Journal of Herpetology, 1:25-36.

GREENE, H.W. 1994. Systematics and natural history, foundations for understanding and conserving biodiversity. American Zoologist, 34:48-56.

KLINK, C.A. \& MACHADO, R.B. 2005. A conservação do Cerrado brasileiro. Megadiversidade, 1:147-155.

KOPP, K.; SIGNORELLI, L \& BASTOS R.P. 2010. Distribuição temporal e diversidade e modos reprodutivos de anfíbios anuros no Parque Nacional das Emas e entorno, estado de Goiás, Brasil. Iheringia, Série Zoológica, Porto Alegre, 100:192-200.

KOVACH, W.L. 2000. MVSP - A multivariate statistical package for Windows, version 3.1. Kovach Computing Services, Penthraeth.

LIMA, A.P.; MAGNUSSON, W.E.; MENIN, M.; ERDTMANN, L.K.; RODRIGUES, D.J.; KELLER, C. \& HÖDL, W. 2006. Guia de Sapos da Reserva Adolpho Ducke, Amazônia Central (Guide to the frogs of Reserva Adolpho Ducke, Central Amazonia). Manaus: Áttema Design Editorial.

LOEBMANN, D. \& HADDAD, C.F.B. 2010. Amphibians and reptiles from a highly diverse area of the Caatinga domain: composition and conservation implications. Biota Neotropica, 10:227-256.

MACEDO, L.C.; BERNARDE, P.S. \& ABE, A.S. 2008. Lagartos (Squamata Lacertilia) em áreas de floresta e de pastagem em Espigão do Oeste, Rondônia, sudoeste da Amazônia, Brasil. Biota Neotropica, 8:133-139.
MESQUITA, D.O. \& COLLI, G.R. 2003. The ecology of Cnemidophorus ocellifer (Squamata, Teiidae) in a neotropical savanna. Journal of Herpetology, 37: 498-509.

MOURA, G.J.B.; FREIRE, E.M.X.; SANTOS, E.M.; MORAIS, Z.M.B.; LINS, E.A.M.; ANDRADE, E.V.E. \& FERREIRA, J.D.C. 2011b. Distribuição geográfica dos répteis do estado do Pernambuco. In: Moura, G.J.B.; Santos, E.M.; Oliveira, M.A.B. \& Cabral, M.C.C. (Eds.), Herpetologia no Estado de Pernambuco. Ministério do Meio Ambiente, Brasília, p.229-290.

MOURA, G.J.B.; SANTOS, E.M.; ANDRADE, E.V.E. \& FREIRE, E.M.X. 2011a. Distribuição geográfica e caracterização ecológica dos anfíbios de Pernambuco. In: Moura, G.J.B.; Santos, E.M.; Oliveira, M.A.B. \& Cabral, M.C.C. (Eds.), Herpetologia no Estado de Pernambuco. Ministério do Meio Ambiente, Brasília, p.50-84.

MYERS, N.; MITTERMEIER, R.A.; MITTERMEIER, C.G.; DA FONSECA, G.A.B. \& Kent, J. 2000. Biodiversity hotspots for conservation priorities. Nature, 403:853-858.

NOGUEIRA, C. \& RODRIGUES, M.T. 2006. The genus Stenocercus (Squamata: Tropiduridae) in Extra-Amazonian Brazil, with the description of two new species. South American Journal of Herpetology, 1:149-165.

NOGUEIRA, C., RIBEIRO, S., COSTA, G.C. and COLLI, G.R. 2011. Vicariance and endemism in a Neotropical savanna hotspot: distribution patterns of Cerrado squamate reptiles. Journal of Biogeography, 38: 1907-1922.

NOGUEIRA, C.; COLLI, G.R. \& MARTINS, M. 2009. Local richness and distribution of the lizard fauna in natural habitat mosaics of the Brazilian Cerrado. Austral Ecology, 34:83-96.

NOGUEIRA, C.; COLLI, G.R.; COSTA, G.C. \& MACHADO, R.B. 2010 Diversidade de répteis Squamata e evolução do conhecimento faunístico no Cerrado. In: Diniz, I.R.; Marinho-Filho, J.; Machado R.B. \&. Cavalcanti, R.B. (Eds.), Cerrado: conhecimento científico quantitativo como subsídio para ações de conservação. Thesaurus editora, Brasília, p.333-375.

OLIVEIRA-FILHO, A.T. \& RATTER, J.A. 2002. Vegetation physiognomies and woody flora of the Cerrado biome. In: Oliveira P.S. \& Marquis, R.J. (Eds.), The Cerrados of Brazil: Ecology and Natural History of a Neotropical Savanna. Columbia University Press, New York, p.91-120.

OLSON, D.M.; DINERSTEIN, E.; WIKRAMANAYAKE, E.D.; BURGUESS, N.D.; POWELL, G.V.N.; UNDERWOOD, E.C.; D'AMICO, J.A.; STRAND, J.C.; LOUCKS, C.J.; ALLNUTT, T.F.; RICKETTS, T.H.; KURA, Y.; LAMOREUX, J.F.; WETTENGEL, W.W.; HEDAO, P. \& KASSEM, K.R. 2001. Terrestrial Ecorregions of the World: A New Map of Life on Earth. Bioscience, 51: 933-938.

PASSOS, D.C., LIMA, D.C. \& BORJES-NOJOSA, D.M. 2011. A new species of Tropidurus (Squamata, Tropiduridae) of the semitaeniatus group from a semiarid area in Northeastern Brazil. Zootaxa, 2930: 60-68.

PASSOS, P.; CARAMASCHI, U. \& PINTO, R.R. 2006. Redescription of Leptotyphlops koppesi Amaral, 1954, and description of a new species of the Leptotyphlops dulcis group from Central Brazil (Serpentes: Leptotyphlopidae). Amphibia-Reptilia, 27:347-357.

PAVAN, D. \& DIXO, M. 2004. A Herpetofauna da área de influência do reservatório da Usina Hidrelétrica Luís Eduardo Magalhães, Palmas, TO Humanitas, 4:13-30.

PAVAN, D. 2007. Assembléias de répteis e anfíbios do Cerrado ao longo do rio Tocantins e o impacto do aproveitamento hidrelétrico da região na sua conservação. Ph.D. Instituto de Biociências da Universidade de São Paulo, São Paulo.

PINHEIRO, L.P. 2010. Similaridade de anfíbios anuros em áreas de Savana Amazônica, Floresta Nacional de Carajás, Pará, Brasil. Dissertation, Universidade Federal do Pará, Pará.

PINNA, P.H.; MENDONCA, A.F.; BOCCHIGLIERI, A. \& FERNANDES, D.S. 2010. A new two-pored Amphisbaena Linnaeus from the endangered Brazilian Cerrado biome (Squamata: Amphisbaenidae). Zootaxa, 2569: 44-54.

RECODER, R. \& NOGUEIRA, C. 2007. Composição e diversidade de répteis Squamata na região sul do Parque Nacional Grande Sertão Veredas, Brasil Central. Biota Neotropica, 7:267-278. 
RECODER, R.S.; TEIXEIRA Jr., M.; CAMACHO, A.; NUNES, P.M.S.; MOTT, T.; VALDUJO, P.H.; GHELLERE, J.M.; NOGUEIRA, C. \& RODRIGUES, M.T. 2011. Répteis da Estação Ecológica Serra Geral do Tocantins, Brasil Central. Biota Neotropica, 11:257-275.

RECODER, R.S.; WERNECK, F.P.; TEIXEIRA Jr., M.; COLLI, G.R. SITES Jr., J.W. \& Rodrigues, M.T. 2014. Geographic variation and systematic review of the lizard genus Vanzosaura (Squamata, Gymnophthalmidae), with the description of a new species. Zoological Journal of the Linnean Society, 171: 206-225.

RIBEIRO, S.C.; FERREIRA, F.S.; BRITO, S.V.; SANTANA, G.G.; VIEIRA, W.L.S.; ALVES, R.R.N. \& ALMEIDA, W.O. 2008. The Squamata Fauna of the Chapada do Araripe, North Eastern Brazil. Caderno de Cultura e Ciência, 3:1-14

RIBEIRO, S.C.; ROBERTO, I.J.; SALES, D.L.; ÁVILA R.W. \& ALMEIDA, W.O. 2012. Amphibians and reptiles from the Araripe bioregion, northeastern Brazil. Salamandra, 48(3):133-146

RODRIGUES MT. 2003. Herpetofauna da Caatinga. In: Leal IR, Tabarelli M, Silva J.M.C. (Eds.) Ecologia e Conservação da Caatinga. Recife, Brazil: Editora Universitária UFPE, Pp 181-236.

RODRIGUES, M.T. 1984a. Notobachia ablephara: novo gênero e espécie do nordeste do Brasil (Sauria, Iguanidae). Papéis Avulsos de Zoologia, 35:361-366.

RODRIGUES, M.T. 1984b. Sobre Platynotus Wagler, 1980, pré-ocupado, substituido por Tapinurus Amaral, 1933, com descrição de uma nova espécie (Sauria, Iguanidae). Papéis Avulsos de Zoologia, 35: 367-373.

RODRIGUES, M.T. 1987. Sistemática, ecologia e zoogeografia dos Tropidurus do grupo torquatus ao sul do rio Amazonas (Sauria, Iguanidae). Arquivos de Zoologia, 31:105-230.

RODRIGUES, M.T. 1988. Distribution of lizards of genus Tropidurus in Brazil (Sauria, Iguanidae). In: W.R Heyer \& P.E. Vanzolini (Eds.) Proceedings of a workshop on neotropical distribution patterns. Pp.305-315. Academia Brasileira de Ciências, Rio de Janeiro, Brasil.

RODRIGUES, M.T. 1996. Lizards, snakes and amphisbaenians of the quaternary sand dunes of the rio Sao Francisco: Bahia: Brazil. Journal of Herpetology, 30(4): 513-523

RODRIGUES, M.T., ANDRADE, G.V. and LIMA, J.D. 2003b. A new species of Amphisbaena (Squamata, Amphisbaenidae) from state of Maranhão, Brazil. Phyllomedusa, 2(1): 21-26.

RODRIGUES, M.T., TEIXEIRA Jr, M., DAL VECHIO, F., AMARO, R.C., NISA, C., GUERRERO, A.C., DAMASCENO, R., ROSCITO, J.G., NUNES, P.M.S., RECODER, R.S. 2013. Rediscovery of the Earless Microteiid Lizard Anotosaura collaris Amaral, 1933 (Squamata: Gymnophthalmidae): A redescription complemented by osteological, hemipenial, molecular, karyological, physiological and ecological data. Zootaxa, 3731: 345-370.

RODRIGUES, M.T.; CAMACHO, A.; NUNES, P.M.S.; RECODER, R.S.; TEIXEIRA JUNIOR, M.; VALDUJO, P.H.; GHELLERE, J.M.B.; MOTT, T. \& NOGUEIRA, C. 2008. A new species of the lizard genus Bachia (Squamata: Gymnophthalmidae) from the Cerrados of Central Brazil. Zootaxa, 1875:39-50.

RODRIGUES, M.T.; PAVAN, D. \& CURCIO, F.F. 2007. Two new species of the genus Bachia (Squamata, Gymnophthalmidae) from Central Brazil. Journal of Herpetology, 41:545-553.

RODRIGUES, M.T.; ZAHER, H. \& CURCIO, F.F. 2001. A new species of lizard, genus Calyptommatus, from the caatingas of the state of Piauí, northeastern Brazil (Squamata, Gymnophthalmidae). Papéis Avulsos de Zoologia, 41(28):529-546.

SANTANA, D.O., DE-CARVALHO, C.B., FREITAS, E.B., NUNES, G.S.S and FARIA, R.G. 2015. First record of Siphonops paulensis Boettger, 1892 (Gymnophiona: Siphonopidae) in the state of Sergipe, northeastern Brazil. Checklist, 11(1): 1531.
SANTANA, G.G.; VIEIRA, W.L.S.; PEREIRA-FILHO, G.A.; DELFIM, F.R.; LIMA, Y.C.C. \& VIEIRA, K.S. 2008. Herpetofauna em um fragmento de Floresta Atlântica no Estado da Paraíba, Região Nordeste do Brasil. Biotemas, 21:75-84.

SICK, H. 1965. A Fauna do Cerrado. Arquivos de Zoologia, 12:71-93.

SILVA JUNIOR, N.J.; SilVa, H.L.R.; RODRIGUES, M.T.; VALLE, N.C.; COSTA, M.C.; CASTRO, S.P.; LINDER, E.T.; JOHANSSON, C. \& SITES JUNIOR, J.W. 2005. A fauna de vertebrados do vale do alto Rio Tocantins em áreas de usinas hidrelétricas. Estudos, 32:57-101.

SILVA, J.F.; FARIÑAS, M.R.; FELFILI, J.M. \& KLINK, C.A. 2006. Spatiaheterogeneity, land use and conservation in the cerrado region of Brazil. Journal of Biogeography, 33:536-548.

SILVA, J.M.C. \& BATES, J.M. 2002. Biogeograhic patterns and conservation in the South American Cerrado: a tropical savanna Hotspot. BioScience, 52:225-233.

SILVEIRA, A.L. 2006. Anfíbios do Município de João Pinheiro, uma área de Cerrado no Nordeste de Minas Gerais, Brasil. Arquivos do Museu Nacional, Rio de Janeiro, 64:131-139.

SILVEIRA, L.F.; BEISIEGEL, B.M.; CURCIO, F.F.; VLDUJO, P.H.; DIXO, M.; VERDADE, V.K.; MATTOX, G.M.T. \& CUNNINGHAM, P.T.M. 2010. Para que servem os inventários de fauna? Estudos avançados, 24:173-177.

TEIXEIRA Jr., M. DAL VECHIO, F.; MOLLO NETO, A. \& RODRIGUES, M.T. 2014. A New Two-Pored Amphisbaena Linnaeus, 1758, from Western Amazonia, Brazil (Amphisbaenia: Reptilia). South Journal of Herpetology, 9(1): 62-74.

TEIXEIRA JR., M.; AMARO, R.C.; RECODER, DAL VECHIO, F \& RODRIGUES, M.T. 2012b. A new dwarf species of Proceratophrys Miranda-Ribeiro, 1920 (Anura, Cycloramphidae) from the highlands of Chapada Diamantina, Bahia, Brazil. Zootaxa, 3551: 25-42.

TEIXEIRA JR., M.; AMARO, R.C.; RECODER, R.S.; SENA, M.A. \& RODRIGUES, M.T. 2012a. A relict new species of Oreobates (Anura, Strabomantidae) from the Seasonally Dry Tropical Forests of Minas Gerais, Brazil, and its implication to the biogeography of the genus and that of South American Dry Forests. Zootaxa, 3158: 37-52.

TORRES-CARVAJAL, O.; ETHERIDGE, R. \& QUEIROZ, K. 2011. A systematic revision of Neotropical lizards in the clade Hoplocercinae (Squamata: Iguania). Zootaxa, 2752: 1-44.

TURCI, L.C.B. \& BERNARDE, P.S. 2008. Levantamento herpetofaunístico em uma localidade no município de Cacoal, Rondônia, Brasil. Bioikos, 22:101-108

TYLOR, E.H. 1968. The Caecilian of the world. A Taxonomic review. University of Kansas Press, Lawrence. Pp 848.

TYLOR, E.H. 1970. Notes on Brazilian Caecilians. The University of Kansas Science Bulletin, 48: 855-860.

VALDUJO, P.H.; CAMACHO, A.; RECODER, R.S.; TEIXEIRA Jr., M.; GHELLERE, J.M.B.; MOTT, T.; NUNES, P.M.S.; NOGUEIRA, C. \& RODRIGUES, M.T. 2011. Anfíbios da Estação Ecológica Serra Geral do Tocantins, região do Jalapão, Estados do Tocantins e Bahia. Biota Neotropica, 11(1): 251-261.

VALDUJO, P.H.; NOGUEIRA, C.C.; BAUMGARTEN, L.; RODRIGUES, F.H.G.; BRANDÃO, R.A.; ETEROVIC, A.; RAMOS-NETO, M.B. \& MARQUES, O.A.V. 2009. Squamate Reptiles from Parque Nacional das Emas and surroundings, Cerrado of Central Brazil. Checklist, 5:405-417.

VANZOLINI, P.E. 1963. Problemas faunísticos do cerrado. In: Simpósio sobre o Cerrado. EdUSP, São Paulo, p.305-321.

VANZOLINI, P.E. 1976. On the lizards of a Cerrado-Caatinga contact: evolutionary and zoogeographical implications (Sauria). Papéis Avulsos de Zoologia, 29:111-119. 
VANZOLINI, P.E. 1988. Distributional patterns of South American lizards. In: Vanzolini P.E. \& Heyer, W.R. (Eds.), Proceedings of a workshop on Neotropical distributional patterns. Academia Brasileira de Ciências, Rio de Janeiro, p.317-342.

VANZOLINI, P.E. 1991. Two further new species of Amphisbaena from the semi-arid Norteast of Brasil (Reptilia, Amphisbaenia). Papéis Avulsos de Zoologia, 37: 347-361.

VAZ-SILVA, W.; GUEDES, A.G.; AZEVEDO-SILVA, P.L.; GONTIJO, F.F.; BARBOSA, R.S.; ALOÍSIO, G.R. \& OLIVEIRA, F.C.G. 2007. Herpetofauna, Espora Hydroelectric Power Plant, state of Goiás, Brazil. Checklist, 3:338-345.

VITT, L.; MAGNUSSON, W.E.; ÁVILA-PIRES, T.C. \& LIMA. A.P. 2008. Guia de lagartos da Reserva Adolpho Ducke, Amazônia Central (Guide to the Lizards of Reserva Adolpho Ducke, Central Amazônia). Manaus, Áttema Design Editorial, P.175.
VITT, L.J.; CALDWELL, J.P.; COLLI, G.R.; GARDA, A.A.; MESQUITA, D.O.; FRANCCA, F.G.R.; SHEPARD, D.B.; COSTA, G.C.; VASCONCELLOS, M.M. \& DE NOVAES E SILVA, V. 2005. Uma atualização do guia fotográfico de répteis e anfíbios da região do Jalapão no Cerrado Brasileiro. Occasional Papers of the Sam Noble Oklahoma Museum of Natural History, $2: 1-24$.

WERNECK, F.P., NOGUEIRA, C., COLLI, G.R., SITES, J.W. and COSTA, G.C. 2012. Climatic stability in the Brazilian Cerrado: implications for biogeographical connections of South American savannas, species richness and conservation in a biodiversity hotspot. Journal of Biogeography, 39 : 1695-1706.

WERNECK, F.P.; COLLI, G.R. \& VITT, L.J. 2009. Determinants of assemblage structure in Neotropical dry forest lizards. Austral Ecolology, 34: 97-115.

WERNECK, F.P.; LEITE, R.N.; GEURGAS, S.R.; RODRIGUES, M.T. 2015. Biogeographic history and cryptic diversity of saxicolous Tropiduridae lizards endemic to the semiarid Caatinga. Evolutionary Biology, 15: 1-24.

Received: 01/09/2015

Revised: 20/06/2016

Accepted: 21/06/2016 